\title{
Motion and equilibrium of a spheromak in a toroidal flux conserver
}

\author{
M. R. Brown, a) D. M. Cutrer, and P. M. Bellan \\ California Institute of Technology, Pasadena, California 91125
}

(Received 27 August 1990; accepted 7 January 1991)

\begin{abstract}
A number of experiments have been performed on spheromaks injected into the empty vacuum vessel of the Caltech ENCORE tokamak (i.e., without tokamak plasma) [Phys. Rev. Lett. 64, 2144 (1990); Phys. Fluids B 2, 1306 (1990) ]. Magnetic probe arrays (in a number of configurations) have been used to make single shot, unaveraged, in situ measurements of the spheromak equilibrium. These measurements are important because (i) they reveal for the first time the equilibrium structure of spheromaks in a toroidal geometry, (ii) they provide a reliable estimate of magnetic helicity and energy of spheromak plasmas used in injection experiments [Phys. Rev. Lett. 64, 2144 (1990)], and (iii) they constitute the first measurements of spheromak motion across and interaction with static magnetic fields (which are useful in corroborating recent theories). Probe measurements in the tokamak dc toroidal field show for the first time that the spheromak exhibits a "double tilt." The spheromak first tilts while in the cylindrical entrance region, emerging into the tokamak vessel antialigned to the dc toroidal field, then expands into the tokamak vacuum vessel, and finally tilts again to form an oblate (nonaxisymmetric, $m=1$ ) configuration. In addition, the spheromak drifts vertically in the direction given by $\mathbf{J}_{\text {eenter }} \times \mathbf{B}_{\text {tok }}$, where $\mathbf{J}_{\text {center }}$ is the unbalanced poloidal current that threads the center of the spheromak torus. Probe arrays at different toroidal locations show that the spheromak shifts toroidally (horizontally left or right) in the direction opposite that of the static toroidal field. In the absence of toroidal flux, the $m=1$ object develops a helical pitch, the sense of the pitch depending on the sign of the spheromak helicity. The spheromak equilibrium in the toroidal vessel is well fit by a pressureless infinite cylindrical model; however, there is evidence of deviation from $m=1$ symmetry because of toroidal effects, nonuniform $J / B$ profile, and finite $\beta$. Experiments performed in a test facility consisting of the spheromak gun and a replica of the entrance region (with a closed end) show that the spheromak is generated with its axis coaxial with that of the gun. Coherent, $m=2$ magnetic modes are observed during the formation stage rotating in the $\mathbf{E} \times \mathbf{B}$ direction at about $125 \mathrm{kHz}$ (rotation velocity corresponding to $40 \%$ of the Alfvén speed).
\end{abstract}

\section{INTRODUCTION}

Spheromaks are toroidal magnetofiuid configurations of the "compact toroid" class (no material linking the center of the torus). Spheromaks are "force-free" in the sense that internal forces (which are largely magnetic $\mathbf{J} \times \mathbf{B}$ forces) balance so that the magnetic fields in the equilibrium are governed by the equation $\nabla \times \mathbf{B}=\lambda \mathbf{B}$, where $\lambda$ is a constant. Recently, spheromaks have been successfully injected into Caltech's ENCORE tokamak ( $R=38 \mathrm{~cm}, a=12 \mathrm{~cm}, I_{p}$ $=2 \mathrm{kA}$, and $B_{\mathrm{tor}}=700 \mathrm{G}$ ) in order to inject helicity and density. ${ }^{1,2}$ An important consideration in these experiments is the magnetic structure and motion of the spheromak in the empty toroidal vacuum vessel (i.e., without tokamak plasma but with dc toroidal field). First, a fit of the in situ magnetic data of the spheromak in the tokamak vessel to a simple model provides an estimate of the amount of magnetic helicity and energy that can be injected into the tokamak. This estimate can then be compared to the helicity increase of the trkamak upon spheromak injection. Second, the dynamics of the spheromak in the dc toroidal field of the tokamak are relevant to theories of spheromak/tokamak interaction. ${ }^{3,4}$ These theories have predictions regarding (i) the drag

\footnotetext{
2) U.S. Department of Energy Fusion Energy Postdoctoral Research Fellow.
}

mechanism responsible for slowing the spheromak as it moves into the tokamak as well as (ii) the orientation of the spheromak in the tokamak magnetic fields. The present measurements are the first of spheromak motion across (i.e., normal to) and interaction with static magnetic fields and for the first time provide a basis of comparison with theory.

The magnetic structure and motion of spheromaks in cylindrical flux conservers have been observed experimentally ${ }^{5}$ and discussed theoretically. ${ }^{6-8}$ These experiments and calculations have shown that a spheromak in an initially axisymmetric ( $m=0$ ) configuration in a cylindrical flux conserver becomes unstable to a tilt when the ratio of its length to radius $(L / r)$ exceeds 1.67. The spheromak then forms a nonaxisymmetric ( $m=1$ ) configuration; this "racetrack" shaped configuration is then stable and persists for a resistive decay time. Schaffer ${ }^{8}$ has further postulated that the $m=1$ shape should develop a helical pitch. Experiments with an " $m=1$ " helicity source" (a kinked $\mathrm{Z}$ pinch not coaxial with the flux conserver) showed that a helical $m=1$ state is formed in a cylindrical entrance region, while an $m=0$ state is formed in a closed flux conserver. Similar observations ${ }^{10}$ and calculations " have been performed for spheromaks in (nearly) spherical fux conservers.

We report here the first experimental measurements of the motion and equilibrium of a spheromak in the dc toroidal magnetic field of a tokamak. These experiments represent 
the first measurements of spheromaks in nonsimply connected flux conservers and the first measurements of spheromaks launched transversely across a magnetic field. Because our spheromak discharge is rather cold $\left(T_{e}<10 \mathrm{eV}\right)$, we have been able to make detailed in situ measurements. Extensive internal measurements of the magnetic structure of the spheromak in the toroidal vessel are possible since a spheromak can be formed externally, then injected into the vessel fitted with probes. Similar measurements in a tokamak discharge ${ }^{1}$ are difficult since large probe arrays in the toroidal vessel make the formation of the tokamak discharge unreliable. Because of this, the measurements presented here are performed without tokamak plasma.

We have used a number of probe arrays and experimental configurations in order to study the motion of the spheromak from formation to equilibrium in the tokamak chamber. Throughout this work we refer to "toroidal" and "poloidal" as directions in the tokamak frame. We will explicitly state when a specific reference is made to spheromak fields (irrespective of external coordinate system). A linear array of 24 discrete magnetic pickup loops measures all components of $\mathbf{B}$ simultaneously at eight locations across the tokamak minor diameter directly in front of the gun [Fig. 1 (a) ]. The probe is sensitive to rapidly varying fields as a result of the injected spheromak and corrections for the dc toroidal field are added numerically. In addition, two quartz-jacketed cross-shaped arrays have been used on both sides of the injection port. Each of the four legs of the crosses have three pairs of loops (to measure the toroidal and poloidal components of the field; see Fig. 2). An eight-sided array has also been used to study the toroidal component of the magnetic field in more detail in the poloidal cross section. The magnetic structure of the spheromak in the entrance region has been studied in a test facility, where the spheromaks were injected into a closed flux conserver identical to the copper entrance region in the injection experiment [Fig. 1(b) ]. In each of the above cases, up to 24 measurements of magnetic field are performed in situ on a single shot.

These measurements show for the first time that the spheromak (i) is initially generated with its axis directed away from the gun, then (ii) tilts while in the cylindrical entrance region and moves into the tokamak vessel with its axis aligned in the direction opposite the tokamak's toroidal field, then finally (iii), while in the tokamak vacuum vessel, tilts again, directing its axis inward toward the axis of the tokamak (again coaxial with the gun). There is also evidence of a vertical drift of the spheromak in the $\mathbf{J}_{\text {center }} \times \mathbf{B}_{\text {tor }}$ direction, where $\mathbf{J}_{\text {center }}$ is the unbalanced poloidal current that threads the center of the spheromak torus. The ultimate configuration is that of an $m=1$ (nonaxisymmetric) magnetic equilibrium. Measurements in the tokamak poloidal cross section at two toroidal locations (to the left and right sides of the spheromak entrance port) show that the spheromak shifts horizontally in the direction opposite that of the applied dc toroidal field. In the absence of toroidal flux the $m=1$ spheromak is observed to develop a helical twist (the sense of twist depends on the helicity sign) and some deviations from $m=1$ symmetry on the inboard side of the tokamak resulting from toroidal effects. Measurements in a replica of the entrance region indicate that the initial generation of the spheromak is coaxial with the gun. An $m=2$ distortion (on the basic $m=0$ magnetic structure) grows and ultimately terminates the discharge.

The paper is organized as follows. In Sec. II, we address some relevant theoretical topics, which provide the framework for our experimental results. We also discuss a simple model for the spheromak equilibrium in the tokamak chamber and review the tilt mode. In Sec. III, we review the Caltech Spheromak Injection Experiment. In particular, the spheromak gun and formation process is discussed, as well
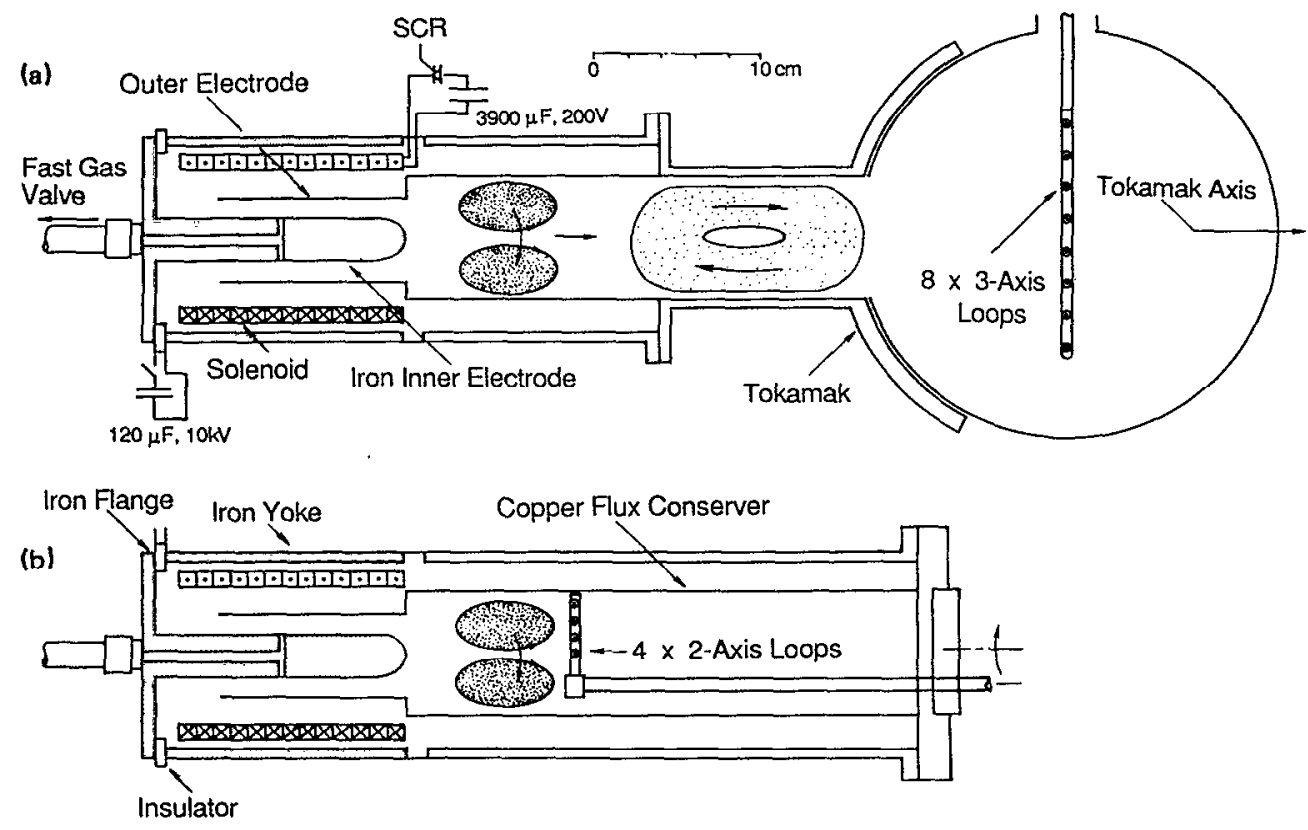

FIG. 1. Schematic representations of probe experiments. (a) Experimental setup showing (left) topology of injected spheromak and (right) spheromak after first tilt. (b) Test stand and probe array used to study the spheromak immediately upon formation. The facility is a replica of the gun and entrance region depicted in (a). 


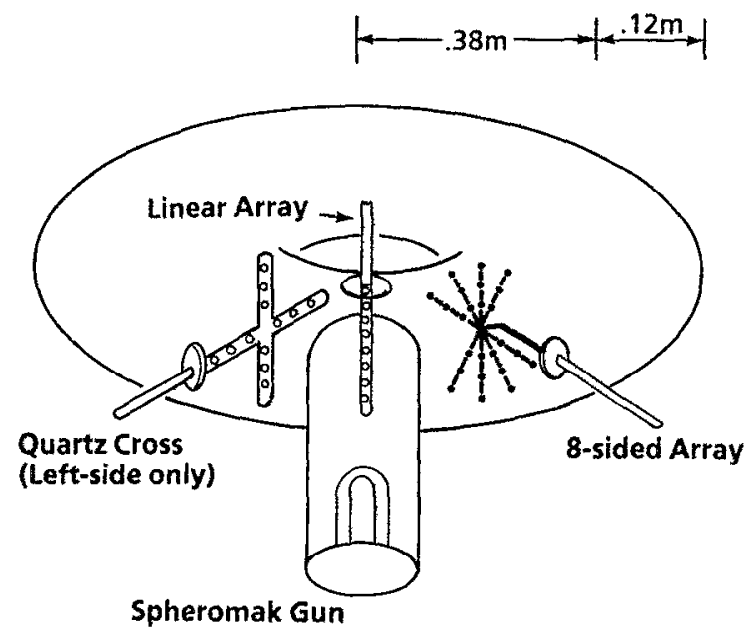

FIG. 2. Schematic showing relative locations of linear probe array, quartz crosses, and eight-legged array. Quartz crosses were placed on both sides of the gun (only the left side is depicted in the figure).

as results from auxiliary diagnostics. In Sec. IV, the results from the linear and poloidal probe arrays are presented. Discussion of spheromak dynamics in tokamak dc toroidal field relevant to theories are presented in Sec. $V$ and a summary is provided in Sec. VI. Measurements from the replica of the entrance region are presented in the Appendix.

\section{THEORETICAL CONSIDERATIONS}

\section{A. Force-free states}

Because spheromaks are detached from external structures, internal forces come to approximate balance and spheromaks are then said to be in a "force-free state." In the low $\beta$ limit, the internal forces are largely magnetic $\mathbf{J} \times \mathbf{B}$ forces so that the force-free condition implies that $\mathbf{J}$ is everywhere parallel to $\mathbf{B}$. We can therefore write the eigenvalue equation governing the spheromak magnetic field structure:

$$
\mathbf{\nabla} \times \mathbf{B}=\lambda \mathbf{B}
$$

since $\nabla \times \mathbf{B}=\mu_{0} \mathbf{J}$. According to the Taylor formalism, ${ }^{12.13}$ $\lambda$ is a constant throughout the spheromak equilibrium. The constant $\lambda$ has a number of interpretations.

First, the quantity $\lambda$ has the units of length ${ }^{-1}$ and is determined by the boundary conditions in which Eq. (1) is solved. For example, in the most simple case of a spheromak coaxial with closed cylinder $^{7}$ ( $m=0$ symmetry), $\lambda=\left(k_{r}^{2}+k_{z}^{2}\right)^{1 / 2}$, where $k_{r}=3.83 / r$ and $k_{z}=\pi / L$, with $r$ and $L$ being the radius and length of the cylindrical flux conserver [ 3.83 is the first zero of $J_{1}(x)=0$ ]. We see that $\lambda$ can be viewed as an eigenvalue of Eq. (1), a geometrical quantity governed by the size of the system.

We can also see from $\mathrm{Eq}$. (1) that $\lambda=\mu_{0} J / B$, or if we multiply numerator and denominator by a suitable area we find $\lambda=\mu_{0} I / \Phi$. This interpretation of $\lambda$ is useful first as a measure of deviations from the "force-free" or Taylor state (a pure force-free state has $\lambda=$ const everywhere). Nonuniform $J / B$ profiles (either peaked or hollow) have been observed to drive low mode number oscillations in several experiments. ${ }^{10,14}$ These oscillations tend to drive the sphero- mak (at least partially) back to a force-free state with uniform $J / B$. In addition, this interpretation is a useful measure of the gun current necessary to overcome the magnetizing flux of a spheromak gun of a given geometry (see Sec. III A).

Finally, we define magnetic helicity $K=\int \mathbf{A} \cdot \mathbf{B} d^{3} x$ and magnetic energy $W=\int \mathbf{B} \cdot \mathbf{B} / 2 \mu_{0} d^{3} x$ (A is the magnetic vector potential). Magnetic helicity is a measure of the linked magnetic flux within a bounded volume and is closely related to field aligned current. Magnetic helicity is a conserved quantity in ideal magnetohydrodynamics (MHD), but it has been postulated ${ }^{12,13}$ (and subsequently verified experimentally ${ }^{15}$ ) that helicity is conserved for times shorter than the resistive decay time even in the presence of turbulent tearing. It can be shown ${ }^{12}$ that if the magnetic energy $W$ is minimized subject to the constraint of constant helicity (using the technique of Lagrange multipliers) the equilibrium magnetic states in a perfectly conducting boundary satisfy Eq. (1) (where $\lambda$ is the Lagrange multiplier). In this interpretation we can write $\lambda=2 \mu_{0} W / K$, where the lowest energy state is that with the lowest value of $\lambda$ (i.e., the largest size or maximum inductance). A spheromak rapidly moving from one region to another of larger dimensions will have a smaller $\lambda$ and therefore less magnetic energy per unit helicity. If the energy and helicity lost to resistive decay is small during the transition then energy is dissipated via turbulence while helicity (fiux linkage) is nearly conserved. The process of shedding magnetic energy while maintaining magnetic helicity in order to regulate $\lambda$ is known as relaxation.

\section{B. Fit of data to model}

We have fit $B_{\text {tor }}$ data (analogous to $B_{z}$ in the cylindrical approximation) from our quartz cross and eight-sided probe arrays to a simple model. The $B_{r}, B_{\theta}$, and $B_{z}$ components of the solution to Eq. (1) in an infinite, perfectly conducting (straight) cylinder can be written ${ }^{8.13}$ (for $m=1$ ) as

$$
\begin{aligned}
B_{r}= & -B_{0} \sin \left(\theta-k_{z} z+\theta_{0}\right) \\
& \times\left(\frac{\lambda+k_{z}}{r v_{k}^{2}} J_{1}\left(v_{k} r\right)-\frac{k_{z}}{v_{k}} J_{0}\left(v_{k} r\right)\right), \\
B_{\theta}= & B_{0} \cos \left(\theta-k_{z} z+\theta_{0}\right) \\
& \times\left(\frac{\lambda+k_{z}}{r v_{k}^{2}} J_{1}\left(v_{k} r\right)-\frac{\lambda}{v_{k}} J_{0}\left(v_{k} r\right)\right), \\
B_{z}= & B_{0} \cos \left(\theta-k_{z} z+\theta_{0}\right) J_{1}\left(v_{k} r\right),
\end{aligned}
$$

where $v_{k}=+\left(\lambda^{2}-k_{z}^{2}\right)^{1 / 2}, \lambda$ is the eigenvalue of Eq. (1), and $k_{z}$ is the axial wave number governing the helical twist. The variable $\theta_{0}$ is simply a coordinate choice that is necessary because the spheromak solution is degenerate with respect to azimuthal rotation. In order to do a least squares fit of the experimental toroidal data, $B_{\text {tor,exp }}$, to the theoretical model we define the quantity

$$
M=\Sigma\left[B_{z, \text { model }}-B_{\text {tor,exp }}\right]^{2} \text {. }
$$

This function is minimized with respect to $k_{z}, \theta_{0}$, and $B_{0}$ through the condition

$$
\nabla M\left(k_{z}, \theta_{0}, B_{0}\right)=0,
$$


or equivalently,

$$
\frac{\partial M}{\partial k_{z}}=0, \quad \frac{\partial M}{\partial \theta_{0}}=0, \quad \frac{\partial M}{\partial B_{0}}=0 .
$$

These three nonlinear equations are solved numerically for the three independent variables $k_{z}, \theta_{0}$, and $B_{0}(\lambda$ is obtained by enforcing the boundary condition, described below).

Since we have assumed a perfectly conducting cylinder, we have the boundary condition that no radial magnetic fiux can exist at the wall of the cylinder, $B_{r}(a)=0$. From Eq. (2), we see that this condition is satisfied when

$$
\left(\lambda+k_{z}\right) J_{1}\left(v_{k} a\right)-a k_{z} v_{k} J_{0}\left(v_{k} a\right)=0 .
$$

Noting that $v_{k}$ is a function of $\lambda$, we again have a nonlinear equation that is solved numerically for $\lambda$ using the value of $k_{z}$ obtained from the least squares fit.

The numerical solution is obtained as follows. An initial guess is made for the values of $\lambda, k_{z}, \theta_{0}$, and $B_{0}$ and each of the four differential equations [ three from the minimization process (4) and one from the boundary condition (5)] is solved separately, giving new values. This procedure is iterated until $M\left(k_{z}, \theta_{0}, B_{0}\right)$ suitably converges and Eq. (5) is solved. For the eight-sided array data, we cannot do a fit in $k_{z}$ because this array only measures $\mathbf{B}$ at one toroidal location; however, good measurements of $k_{z}$ were obtained from the quartz cross data fits.

The boundary condition [Eq. (5)] also shows that spheromaks with positive and negative helicity have opposite senses of twist. We definc the helicity sign as positive $(\lambda>0)$ if current flows parallel to the magnetic field (righthanded spheromak) and negative $(\lambda<0)$ if antiparallel (left-handed spheromak). We can rewrite Eq. (5) as

$$
\lambda / k_{z}=a v_{k} J_{0}\left(v_{k} a\right) / J_{1}\left(v_{k} a\right)-1 \text {. }
$$

Since changing the helicity sign of the spheromak corresponds to changing the sign of $\lambda$, we wish to see the behavior of (6) under the transformation $\lambda \rightarrow-\lambda$. Since $v_{k}$ is a function of $\lambda^{2}$ and $k_{z}^{2}$ only, the right-hand side of (6) does not change sign when the sign of $\lambda$ changes. Clearly, in order for (6) to be satisfied for all $\lambda$, a sign change in $k_{z}$ must accompany a sign change in $\lambda$. Therefore this model predicts that left- and right-handed spheromaks should have opposite senses of twist. This prediction is verified experimentally (see Sec. IV B).

Using the values of $\lambda, k_{z}, \theta_{0}$, and $B_{0}$, the magnetic energy $\left(W_{\text {mag }}=\int \mathbf{B} \cdot \mathbf{B} / 2 \mu_{0} d^{3} x\right)$ was calculated. The fit parameters give the component values of the magnetic field at all points in the cylinder. The size of the spheromak was estimated from experimental data, which showed that the spheromak has length/radius $\cong 4$ (see Sec. IV B, Figs. 11 and 12). By dividing the cylinder into volume elements ( $r d r d \theta d z$ ), evaluating $\mathbf{B} \cdot \mathbf{B}=B_{r}^{2}+B_{\theta}^{2}+B_{z}^{2}$ at a point in each element, and numerically summing over all the volume elements, we obtain $\int \mathbf{B} \cdot \mathbf{B} d^{3} x$. Dividing this quantity by $2 \mu_{0}$ gives $W_{\text {mag }}$, and dividing it by $\lambda$ gives the magnetic helicity $\left(K_{\mathrm{ph}} \equiv \int A \cdot B d^{3} x=2 \mu_{\mathrm{o}} W_{\mathrm{mag}} / \lambda\right)$.

This model could be made more sophisticated by (1) considering the variation of $\lambda$ with the poloidal flux function $(\Psi)$ as other groups have done. ${ }^{14}$ To first order, this correction can be expressed as $\lambda(r)=\lambda+\alpha r$, where $\alpha$ is a con- stant to be determined by least squares minimization. (2) Additionally, one could account for toroidal effects of the flux conserver by letting $B_{z}$ be a linear function of the tokamak major radius [i.e., $B_{z}=\alpha(R+r \sin \theta) B_{z}(r, \theta, z)$, where $R$ is the major radius].

\section{Tilt instability}

The most striking dynamical feature of the spheromak is its instability to the tilt mode. The tilt mode in a cylindrical flux conserver is characterized by a $90^{\circ}$ rotation of the spheromak axis (magnetic moment) from being coaxial with its cylindrical container to being normal to the cylindrical axis (see Fig. 3). The axisymmetric (coaxial) configuration has $m=0$ symmetry (where magnetic fields vary like $e^{i m \theta}$ with $\theta$ the azimuthal or poloidal angle), whereas the nonaxisymmetric configuration has $m=1$ symmetry. The instability can be understood from the following simple energy argument.

In Fig. 3, we depict a spheromak in a cylindrical fiux conserver with $m=0$ symmetry [Fig. 3(a)] and with $m=1$ symmetry [Fig. 3 (b)]. We assume that the poloidal flux and the total volume is the same for both cases. We can estimate the magnetic energy in each case by first calculating the magnetic field (flux density). For the $m=0$ case [Fig. $3(\mathrm{a})], \quad B_{\mathrm{pol}} \cong \Phi_{\mathrm{pol}} /\left[\pi(r / 2)^{2}\right] \quad\left(\Phi_{\mathrm{pol}}\right.$ threads a circle), whereas for the $m=1$ case [Fig. 3(b)], $B_{p o l}$ $\cong \Phi_{\mathrm{pol}} /[\pi(r / 2)(L / 4)]$ ( $\Phi_{\mathrm{pol}}$ threads an ellipse $)$. We find that the magnetic field (and therefore the magnetic energy, $\left.W_{\text {mag }}=B^{2} / 2 \mu_{0}\right)$ is lower for the $m=1$ case if $L>2 r$. More sophisticated analysis ${ }^{6,7}$ shows instability if $L>1.67 r$. The physical picture is that the poloidal flux (which is conserved in ideal MHD) can spread out over a larger area in the $m=1$ configuration so that the spheromak tilts from the $m=0$ to the lower energy $m=1$ configuration if it becomes taller than it is wide. We observe our spheromak undergoing two tilts, once in the cylindrical entrance region, then again in the nearly cylindrical tokamak vessel.

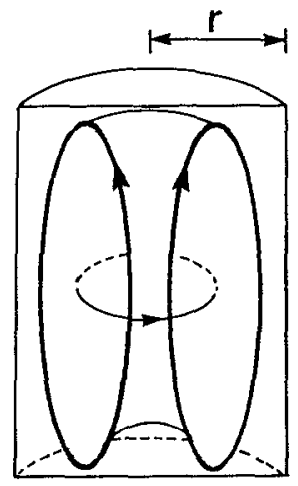

$B_{1} \cong \Phi / n(r / 2)^{2}$

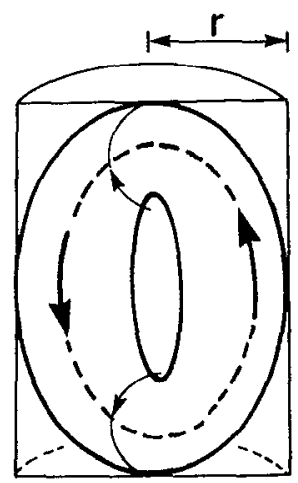

$B_{2} \cong \Phi / \Pi(r / 2)(L / 4)$

$$
\mathrm{B}_{2}<\mathrm{B}_{1} \text { if } \mathrm{L}>2 \mathrm{r}
$$

FIG. 3. Tilt instability. Note that typical poloidal fields $B_{2}$ in the figure on the right are less than $B_{1}$ if $L>2 r$. More sophisticated analysis shows instability when $L>1.67 r$. 


\section{EXPERIMENTAL APPARATUS}

\section{A. Spheromak gun}

The spheromak is formed by a coaxial, magnetized plasma gun similar in design to those used by Alfvén ${ }^{16}$ (Fig. 1). The center electrode of the gun and an outer yoke (constructed of high permeability cold rolled steel and Monel) form a magnetic circuit. The center electrode is $2.5 \mathrm{~cm}$ in diameter, the outer electrode is $5.1 \mathrm{~cm}$ in diameter and the cylindrical entrance region is $7.6 \mathrm{~cm}$ in diameter. When an external solenoid is energized, magnetic flux is concentrated in the circuit and flows radially from the tip of the center electrode (called "stuffing flux"). Hydrogen gas is puffed into the gap between the center electrode and an outer electrode. A high voltage capacitor bank is then discharged through the gas forming a radial current sheet. An axial $\mathbf{J} \times \mathbf{B}$ force accelerates the current sheet along the gun barrel until the plasma reaches the magnetic flux at the gun muzzle. If the $\mathbf{J} \times \mathbf{B}$ force is strong enough to overcome the magnetic tension at the gun muzzle, then a free spheromak forms and propagates away from the gun.

We are able to generate spheromaks of either helicity sign. Operationally, we change the spheromak helicity sign by reversing the direction of the stuffing flux (while maintaining the polarity of the electrodes). If the stuffing flux flows inward (toward the center electrode) and negative voltage is applied to the center electrode (so that the gun current also flows inward) then a positive helicity spheromak is formed. We could also reverse the polarity of the electrodes while maintaining the direction of the stuffing fiux but this requires reconfiguring our high voltage supply and so is not practical. We have operated with both polarities but found that the gun performs more reliably with a negative center electrode. The characteristics of spheromaks generated with either polarity electrode are similar (i.e., a righthanded spheromak generated from a positive center electrode with outflowing stuffing flux has the same properties as a right-handed spheromak generated from a negative center electrode with inflowing stuffing fux).

Spheromak formation with the coaxial magnetized plasma gun is analogous to the formation of water drops with an eye dropper. The magnetic tension of the "stuffing flux" is analogous to the surface tension of the drop and the magnetic pressure (due to the $\mathbf{J} \times \mathbf{B}$ force at the back of the gun) is analogous to the pressure applied at the bulb of the eye dropper. It is well known that there exists a threshold (dependent on the size of the dropper) on the amount of pressure required to overcome the surface tension of the drop. If enough pressure is applied and the threshold is exceeded, then a free drop is formed (after undergoing a nonlinear process to break the surface tension). Similarly, there is an operational threshold (dependent on the size of the gun) on the amount of magnetic pressure (parametrized by $I_{\text {gun }}$ ) required to overcome the magnetic tension at the end of the gun (parametrized by $\Phi_{\text {gun }}$ ). Once the threshold is exceeded, then a free spheromak is formed (after undergoing a nonlinear tearing and reconnection process to break the magnetic tension). If not enough pressure is applied to the bulb then the drop merely distends but does not break free of the dropper.
If too much pressure is applied to the bulb (the limit of small surface tension), then a stream of water is generated. Similarly, if we do not apply the "stuffing flux," then firing the gun produces a stream of unconfined plasma (this is the conventional Marshall gun operation).

We use a $10 \mathrm{kV}, 6 \mathrm{~kJ}$ fast ( $10 \mu \mathrm{sec}$ rise time) capacitor bank to break down the gas in the gun and form the radial current sheet. A slow ( $2 \mathrm{msec}$ rise time), low energy ( $100 \mathrm{~J}$ ) capacitor bank is used to energize the solenoid, which provides the gun flux, $\Phi_{\text {gun }}$ (up to $1 \mathrm{mWb}$ ). The gun flux is defined as the flux passing through the center electrode at the location of the gas ports (halfway down its length) and is measured with a flux loop encircling the center electrode. We typically operate with $\Phi_{\text {gun }}=0.4 \mathrm{mWb}$ and discharge current, $I_{\text {gun }}=100 \mathrm{kA}$, with a $7 \mathrm{kV}$ charge on the capacitor. We have found it is necessary to operate above a gun threshold (as mentioned above), $\lambda_{\mathrm{th}}=\mu_{0} I_{\text {gun }} / \Phi_{\text {gun }} \cong 200$ $\mathrm{m}^{-1} \cong \pi / \delta_{\text {gap }}$ (where $\delta_{\text {gap }}$ is the radial distance between inner and outer electrodes) otherwise a free spheromak is not formed (Barnes et al. ${ }^{15,17}$ have made similar observations on the CTX spheromak). A gas puff from a fast acting solenoid valve (LANL design ${ }^{18}$ ) provides a peak pressure in the gun breech of about 0.1 Torr. This gas is fully ionized by the discharge current. The spheromak moves from the gun to the flux conserver at the local Alfvén velocity (about $3 \times 10^{4}$ $\mathrm{m} / \mathrm{sec}$ ).

\section{B. Auxillary diagnostics}

A double Langmuir probe is used to measure the density and temperature of the spheromak as it enters the tokamak chamber. The probe is located in the center of the tokamak vessel directly in front of the gun (about $30 \mathrm{~cm}$ from the gun muzzle). The tips of the probe consist of $0.060 \mathrm{in} .(1.5 \mathrm{~mm})$ tungsten rod (exposed about $2 \mathrm{~mm}$ and separated $2 \mathrm{~mm}$ ). A capacitor switched by an SCR electrically connects the probe tips so that when the spheromak plasma passes the probe, the capacitor discharges through the plasma between the tips. The current that flows is limited by ion flow at the sound speed. We typically draw 10-40 A of probe current. By varying the voltage on the capacitor from shot to shot, the electron temperature can be measured according to standard double Langmuir probe theory. ${ }^{19}$ We measure densities up to $10^{15} \mathrm{~cm}^{-3}$ and temperatures of about $7 \mathrm{eV}$ using this technique. Finally, these density and electron temperature measurements imply short collision times. Specifically, the electron-ion thermal equilibration time is about $1 \mu \mathrm{sec}$ for $n_{e}=10^{15} \mathrm{~cm}^{-3}$ and $T_{e}=7 \mathrm{eV}$, so that in the typical 20 $\mu \mathrm{sec}$ lifetime of the spheromak we expect $T_{i} \cong T_{e}$. In Fig. 4 we present typical plots of gun current (using Rogowski loop), spheromak magnetic field (from a loop in the quartz cross array) and spheromak density (from the double Langmuir probe).

The density measurements from the Langmuir probe are consistent with microwave interferometric measurements performed at the opposite side of the tokamak chamber. Using a $3 \mathrm{~mm}$ interferometer, we measure plasma densities up to $10^{13} \mathrm{~cm}^{-3}$ after plasma expansion fills the entire tokamak vessel. The ratio of tokamak volume to the initial spheromak volume is about 100:1 so we conclude that 


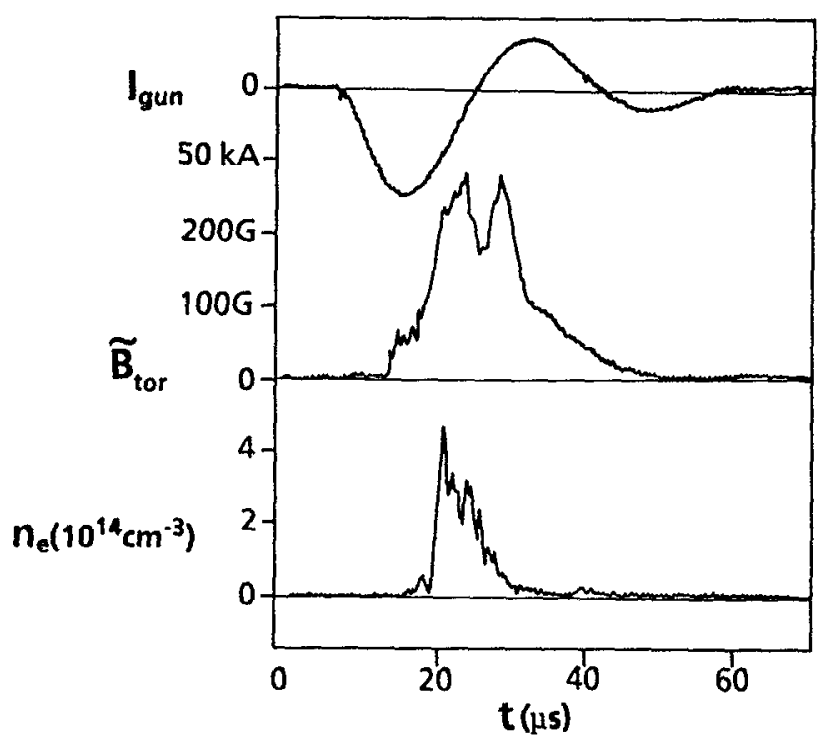

FIG. 4. Typical traces of gun current (measured with a Rogowski loop), spheromak magnetic field (toroidal component from quartz cross), and plasma density (from double Langmuir probe).

the initial spheromak density was about $10^{15} \mathrm{~cm}^{-3}$. This estimate is a lower bound since plasma can be lost due to recombination, charge exchange, and toroidal drift loss. Finally, the density measurements are consistent with complete ionization of the neutral hydrogen puffed into the gun breech.

We have also made direct measurements of the plasma $\beta=2 \mu_{0} n\left(T_{c}+T_{i}\right) / B^{2}$ with a diamagnetic loop, which encircles the tokamak poloidal cross section. The injected spheromak displaces some of the static, applied toroidal flux (see sketch in Fig. 5) and thereby induces a voltage in the loop. It can be shown ${ }^{20}$ with simple assumptions $(\beta \ll 1$ and $n_{e}=n_{0} e^{\left.-\left(r / r_{1}\right)^{2}\right)}$ that $\Delta \Phi=\int V_{\text {diamag }} d t \cong \pi a^{2} \beta B_{0} / 2$, where $a$ is the minor radius, $B_{0}$ is the applied toroidal field, and $V_{\text {diamag }}$ is the voltage induced on the loop. From these measurements we find a $\beta \cong 2 \Delta \Phi / \Phi_{0}$ of about $5 \%$ to $10 \%$. It should be noted that this is not a precise measurement but provides an independent estimate of plasma properties.

\section{MAGNETIC PROBE ARRAY RESULTS}

\section{A. Linear array results}

The spheromak has been injected into the de toroidal field of the ENCORE tokamak without tokamak plasma $\left(B_{\text {tor }}=170 \mathrm{G}\right)$. Measurements were made of the fluctuating spheromak fields in the presence of the applied tokamak toroidal field using a linear array of 24 electrostatically shielded magnetic pickup loops. The loops were oriented to measure $B_{2}, B_{\theta}$, and $B_{\phi}$ (in the tokamak frame) at eight collinear locations separated by $2 \mathrm{~cm}$. Each identical tenturn loop is about $0.5 \mathrm{~cm}$ in diameter and the arrays are covered with a 0.001 in. thick slit copper electrostatic shield. The array is covered with a $9 \mathrm{~mm}$ o.d. quartz jacket to allow rapid penetration of magnetic flux. Probe signals from each loop are integrated with identical precision passive integrator networks (integration time is $100 \mu \mathrm{sec}$ ). Signals are re- corded with 24 identical channels of $10 \mathrm{MHz}, 8$ bit digitizers. The probe axis is placed vertically along a minor diameter of the tokamak directly in front of the spheromak gun (see Fig. 1).

The picture that emerges from these measurements is depicted in the sketch in Fig. 5. We know that the spheromak is generated with its axis coaxial with the gun (from measurements in the replica of the entrance region; see the Appendix) [Fig. 5(a)]. We then infer that the spheromak tilts in the entrance region [some time before we first see a signal on the linear array; Fig. 5(b) ]. At about 6-10 $\mu \mathrm{sec}$, our first view of the spheromak is coaxial with the tokamak "cylinder" (i.e., with the spheromak axis normal to the axis of the gun). The spheromak at this time is oriented with its magnetic moment antialigned with the dc applied toroidal field. Finally, the spheromak tilts so that it is no longer axisymmetric with the tokamak vessel (but again is coaxial with the gun). After this direct measurement of the second tilt, the spheromak is observed to drift vertically in the tokamak vessel (at about 14-19 $\mu \mathrm{sec}$ ). Experimental evidence for this picture is presented in Fig. 6.
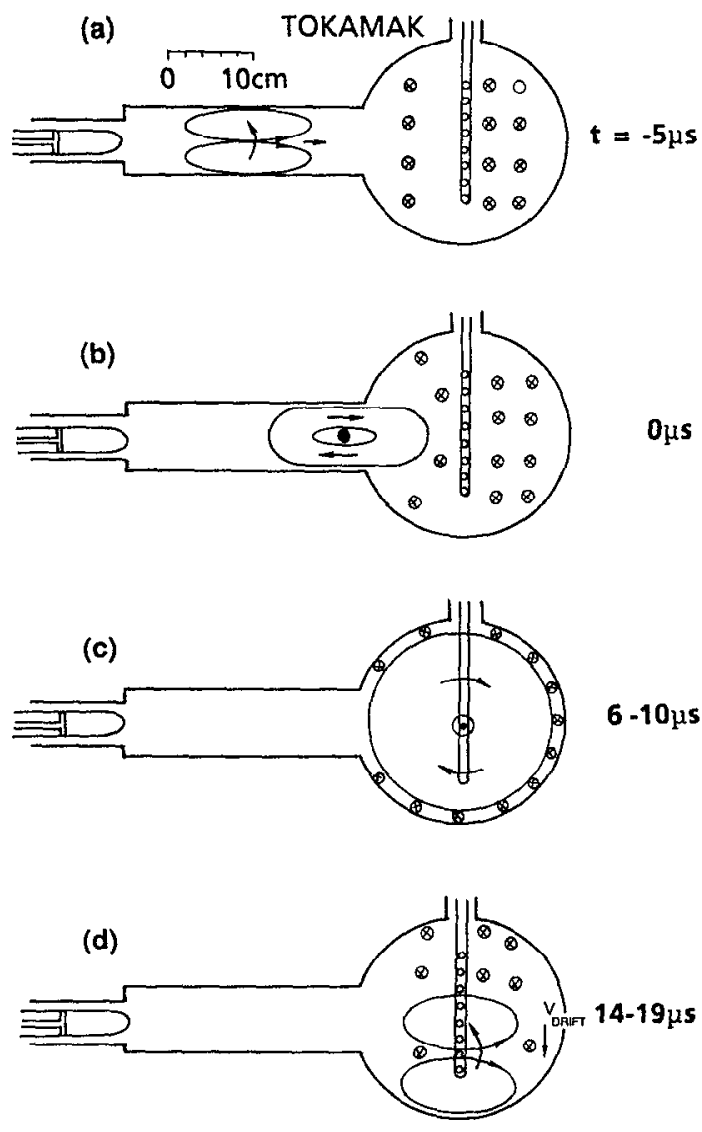

FIG. 5. Motion of spheromak. (a) Spheromak initially formed coaxial with gun (a left-handed spheromak is shown); (b) the spheromak tilts while in the cylindrical entrance region ( $m=1$ symmetry with respect to the entrance region cylinder) and begins to displace the applied tokamak toroidal field; (c) the spheromak has moved into the tokamak vessel $(m=0$ symmetry with respect to the tokamak vessel) and is antialigned with the tokamak field; (d) the spheromak drifts vertically in tokamak vessel in $\mathbf{J}_{\text {cemer }} \times \mathbf{B}_{\text {tok }}$ direction, where $\mathbf{J}_{\text {cemter }}$ is the unbalanced spheromak poloidal current threading its center (directed to the left in the figure) and $B_{t+k}$ is the tokamak toroidal field (directed into the page). 

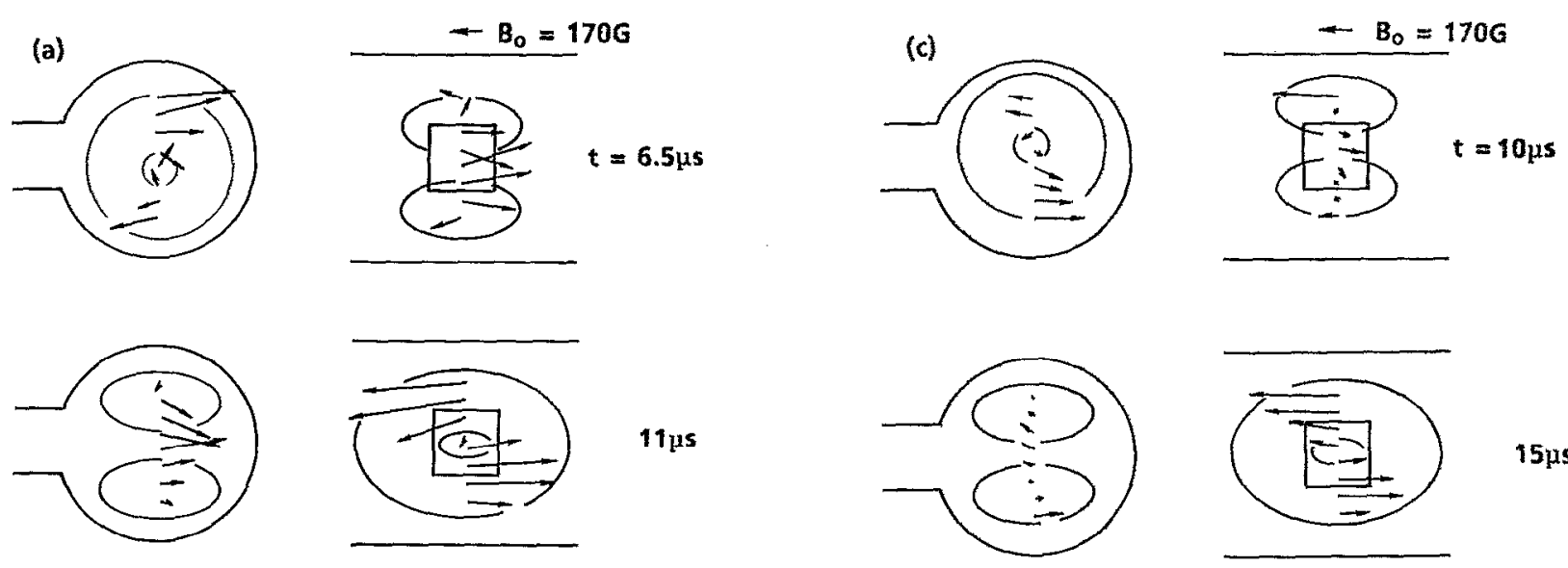

$15 \mu \mathrm{s}$
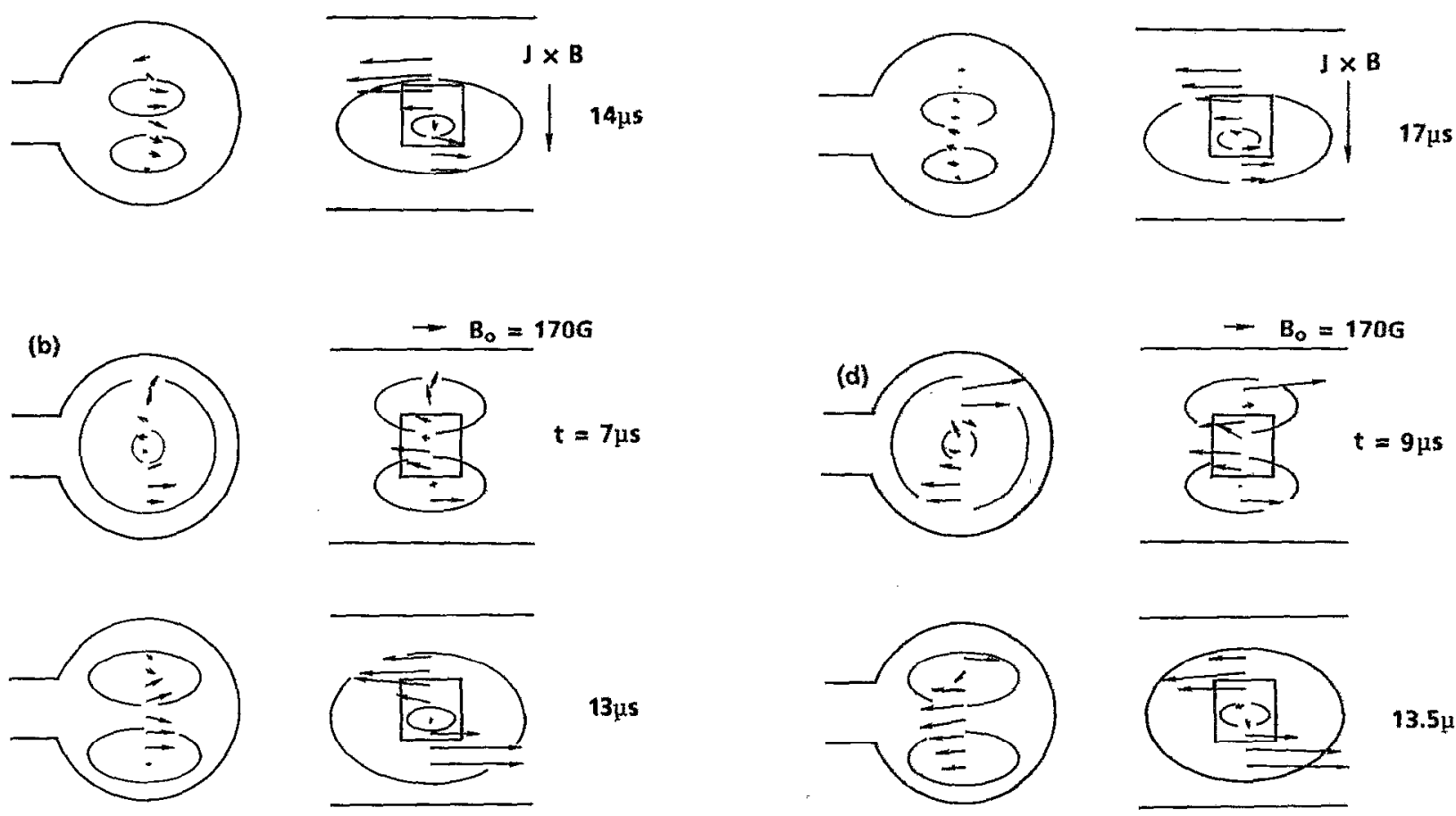

$13.5 \mu \mathrm{s}$
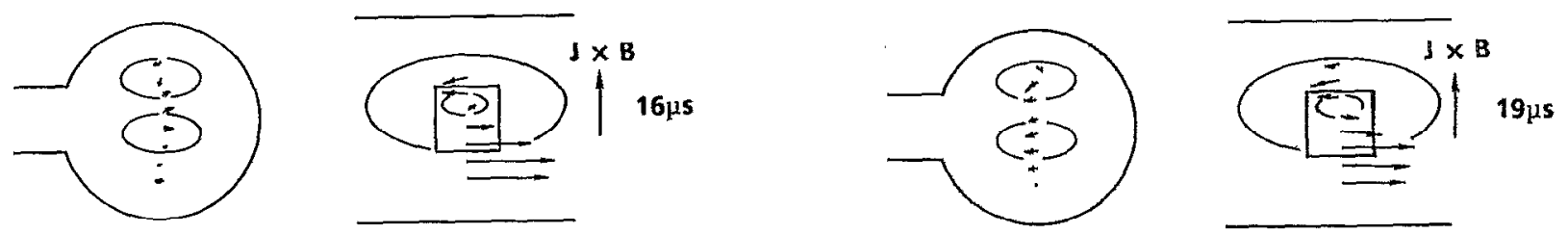

FIG. 6. Data from linear array showing initial $m=0$ symmetry (after first tilt) and antialignment, second tilt to $m=1$ symmetry, and vertical drift. Left side of each figure depicts $B_{\text {pul }}$ and $B_{z}$ viewing along the tokamak toroidal direction (where the spheromak enters from the left). Right side of each figure depicts $B_{t, r}$ and $B_{z}$ as viewed from the perspective of the gun. Magnitude of applied toroidal field is $170 \mathrm{G}$ (no tokamak plasma). The scale (indicated by the $170 \mathrm{G}$ arrow) is the same for all figures (longest arrows represent $600 \mathrm{G}$ ). Here $I_{\text {gun }}=90 \mathrm{kA}$ and $\Phi_{\mathrm{gum}}=0.4 \mathrm{mWb}$. (a) A left-handed spheromak injected into a left-going toroidal field; (b) a left-handed spheromak into a right-going toroidal field; (c) a right-handed spheromak into a left-going toroidal field; and (d) a right-handed spheromak into a right-going toroidal field.

Sequences of measurements are presented in Figs. 6(a)6(d). The diagram on the left of each panel depicts spheromak magnetic fields in a tokamak minor cross section (where the spheromak enters from the left), i.e., $B_{\text {pol }}$ and $B_{z}$ in the tokamak frame are presented (the point of view is along the tokamak toroidal direction). The diagram on the right of each panel depicts a view of the tokamak from the point of view of the spheromak gun (the applied dc $B_{\text {tor }}$ is horizontal and $B_{\text {tor }}$ and $B_{z}$ are presented, the square represents the entrance port). Data are presented of both left- 
handed and right-handed spheromaks injected into either left-going or right-going dc $B_{\text {tor }}$. In each of the four permutations, the magnitude of the applied $B_{\text {tor }}$ is $170 \mathrm{G}$ and time is measured from the arrival of the spheromak at the probe array (the spheromak typically takes $10 \mu$ sec to traverse the $30 \mathrm{~cm}$ from gun to probe array). The data in each column are each from a single shot (without smoothing or averaging) and the length of each arrow (from base to tip) is proportional to the measured magnetic field. The dimensions depicted are drawn to scale (scale given by length of the $170 \mathrm{G}$ arrow at the upper right is the same for all the following figures). The capacitor bank voltage is $6 \mathrm{kV}, I_{\text {gun }}=90 \mathrm{kA}$, and $\Phi_{\text {gun }}= \pm 0.4 \mathrm{mWb}$ for this sequence.

Each column depicts the following three step sequence. The first frame shows that the spheromak has moved into the tokamak fields with its major axis (defined by its magnetic moment) directed antiparallel to the toroidal field of the tokamak. The spheromak settles into this configuration after about 6-10 $\mu \mathrm{sec}$ (this is our first view of the spheromak after formation). This orientation constitutes a $90^{\circ}$ tilt from its initial configuration in the gun and entrance region (we have verified that the spheromak is in fact generated with its axis coaxial with the gun; see the Appendix). At this early stage, the spheromak is in an axisymmetric, $m=0$ configuration. Recall that a spheromak in a cylindrical flux conserver is unstable to a tilt when ${ }^{6,7}$ the ratio of its length to its radius satisfies the condition, $L / r \geqslant 1.67$. Evidently, as the spheromak expands away from the gun and while it is still in the long, narrow cylindrical entrance region, the $L / r$ ratio exceeds 1.67 and the spheromak tilts (into the orientation expected for a diamagnetic object; see the discussion and Fig. 8 below). Note that the spheromak magnetic moment is antialigned with the tokamak toroidal field in every case; the magnetic moment is directed to the right where the applied field is directed to the left [Figs. 6(a) and 6(c)], and to the left where the applied field is to the right [Figs. $6(\mathrm{~b})$ and 6(d)].

The next frame in each column shows that the spheromak has tilted again (into an $m=1$ configuration) so that its axis is directed inward (toward the tokamak axis) in the case of the left-handed spheromak or outward (away from the tokamak axis) in the case of the right-handed spheromak. In other words, we can now look through the hole in the center of the spheromak torus by looking from the point of view of the gun, whereas, at earlier times, our eye needed to be directed along the tokamak toroidal field. This later stage is evident at about 11-15 $\mu \mathrm{sec}$. It appears, then, that the $m=0$ spheromak expands along the tokamak toroidal field, again becomes prolate $(L / r \geqslant 1.67)$ and tilts again to the $m=1$ configuration. We refer to this phenomenon as a "double tilt." It should be noted that the applied toroidal field has little effect on the spheromak dynamics to this point. One might imagine that the applied field could prevent the second tilt, but evidently the internal MHD effects dominate external torque effects. Note that in each sequence [Figs. 6(a) $-6(d)$ ] the toroidal field of the spheromak is directed in the counterclockwise sense. This is the same sense in which the spheromak was initially generated (consistent with the negative center electrode). In some cases, the spher- omak's ultimate configuration is $180^{\circ}$ reversed from its initial configuration (see Fig. 7). The spheromak remains in this configuration and decays with an $e$-folding time of 20 $\mu \mathrm{sec}$. The spheromak lifetime is apparently not significantly affected by the presence of the linear probe. We have retracted the probe so that only a few centimeters are exposed to spheromak plasma and observed approximately the same lifetime.

The last frame in each column shows that late in the decay phase the spheromak has drifted vertically in the tokamak vessel. The drift is evident by noting the location of the spheromak axis (the change in direction of the spheromak toroidal field on the right-hand panel). In Fig. 6(a), note that the spheromak axis moves down from the fourth loop from the top in the second panel to the fifth loop in the third panel. The direction of the drift is given by $\mathbf{J}_{\text {center }} \times \mathbf{B}_{\text {tor }}$, where $\mathbf{J}_{\text {center }}$ is the poloidal current threading the spheromak torus (and $\mathbf{J}_{\mathrm{pol}}$ is the current that generates the spheromak toroidal field). We have generated spheromaks using both positive and negative center electrodes, with both left-handed and right-handed helicity and with both clockwise and counterclockwise orientation of toroidal field in the final $m=1$ configuration; in each of the eight permutations of these cases, we find a drift in the $\mathbf{J}_{\text {center }} \times \mathbf{B}_{\text {tur }}$ direction. In each case, the spheromak has the same $m=1$ symmetry but is displaced about $2 \mathrm{~cm}$ vertically in about $2-5 \mu \mathrm{sec}$ (at about 14-19 $\mu \mathrm{sec}$ after the spheromak arrives at the probe array).

It is clear that the force responsible for the motion is of the form $\mathbf{Y} \times \mathbf{B}$ (where $\mathbf{Y}$ is some vector quantity), where the force must be integrated over the volume. The drift direction is normal to $\mathbf{B}$ and when the direction of the static toroidal magnetic field is reversed, the equilibrium has the same topology, but is observed to drift in the opposite direction. We can rule out $q \mathbf{v} \times \mathbf{B}$ forces since $q \mathbf{v} \times \mathbf{B}$ is in the direction opposite the observed drift. Here, $\mathbf{v}$ is the inward directed velocity of the spheromak as it moves into the chamber from the gun and $q$ is the unbalanced charge. In addition, although the space potential of the spheromak is rather high ( $\cong 1 \mathrm{kV}$ ), there is not enough charge imbalance for the vertical displacement to be attributed to a $\mathbf{v} \times \mathbf{B}$ force. Also, the inward velocity has been reduced to nearly zero while the drifting persists. Similarly, generalized $\mathbf{F} \times \mathbf{B}$ drifts can be ruled out because the observed drift velocity did not decrease with increasing $\mathbf{B}$. The motion is probably due to an unbalanced $\mathbf{J} \times \mathbf{B}$ force since only about $100 \mathrm{~A}$ of unbalanced current is sufficient to account for the observed motion (i.e., a vertical acceleration from rest to the observed drift velocity of about $10^{6} \mathrm{~cm} / \mathrm{sec}$ in a few $\mu \mathrm{sec}$ ) and this magnitude of current has been measured in ground paths.

This $\mathbf{J} \times \mathbf{B}$ hypothesis suggests the possibility of current flow along unbroken field lines from the gun threading through the spheromak. This is unlikely, however, because the current in the gun has rung through zero while the drift is observed. In addition, the "flipped" spheromaks (discussed with Fig. 7 below) are strong evidence that our spheromaks are magnetically completely detached from the gun.

In Fig. 7, we present a sequence in the same format as in Figs. 6(a) -6 (d). This sequence is the same as that in Fig. 6(b) (left-handed spheromak generated from a gun with 

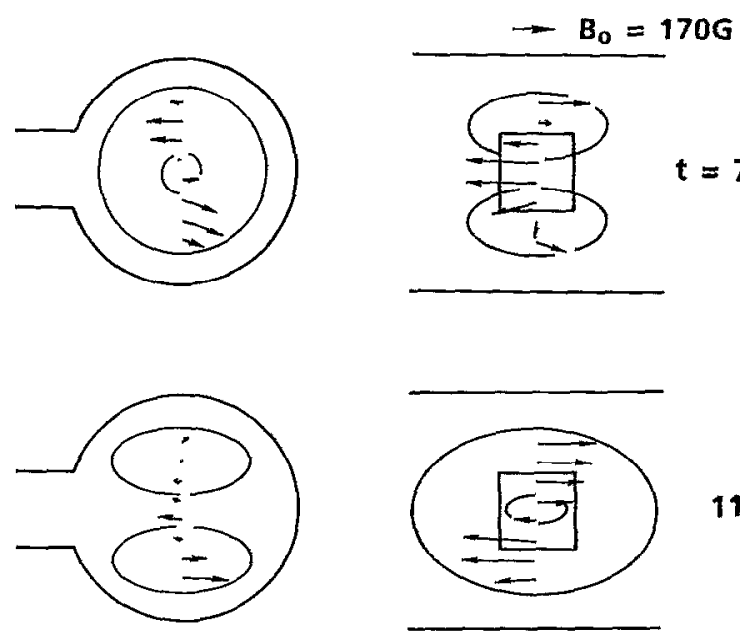

$t=7 \mu \mathrm{s}$
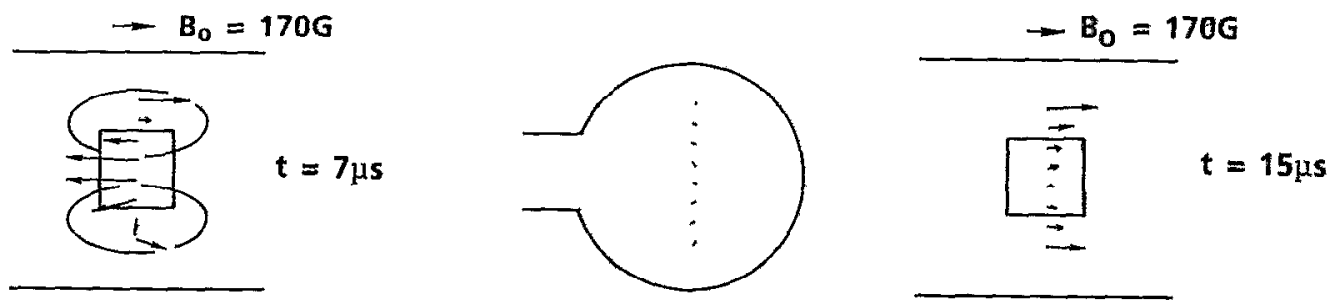

FIG. 8. Shot with stuffing flux disabled $\left(\Phi_{\mathrm{g}_{111}}=0\right.$, Marshall gun shot). Note the diamagnetic effect of the dense plasma displacing the applied field.

$11 \mu \mathrm{s}$
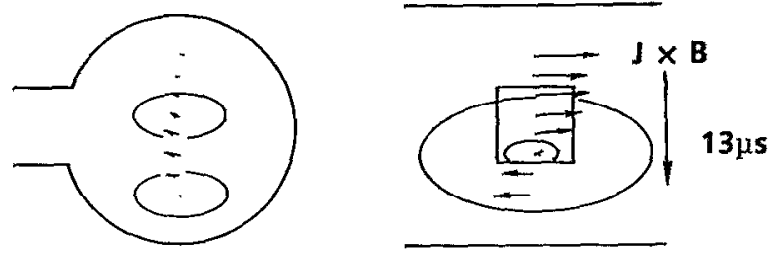

FIG. 7. The same as Fig. 6(b), except with clockwise orientation of the spheromak toroidal field and therefore opposite $J_{\text {semer }}$ and drift direction.

negative center electrode injected into a right-going $B_{\text {tor }}$ ), except that the $m=1$ configuration has its toroidal field directed clockwise (as opposed to counterclockwise). Note that in this case $\mathbf{J}_{\text {center }}$ is directed inward and $B_{\text {tor }}$ is to the right so that $\mathbf{J} \times \mathbf{B}$ is directed downward (in the direction of the observed drift). This is an instance where the ultimate configuration of the spheromak is $180^{\circ}$ reversed from the orientation it was initially generated. In other words, the spheromak is generated with its axis directed away from the gun, then tilts so that its axis is directed $90^{\circ}$ from the axis of the gun, then finally tilts another $90^{\circ}$ so that its axis is generated back toward the gun. This is evidence that the spheromak has magnetically disconnected from the gun (it is unlikely that there are unbroken field lines extending from the gun, wrapping around the spheromak, and threading the hole of the torus from the back).

In Fig. 8, we present data from the linear array from a shot without stuffing flux ( $\Phi_{\mathrm{gun}} \cong 0$, conventional Marshall gun operation $\left.{ }^{21}\right)$. We fire the unmagnetized gun into the magnetized tokamak vessel as before $\left(B_{\text {tor }}=170 \mathrm{G}\right.$ right going). The effect of injecting a stream of dense, unmagnetized plasma into a static magnetic field is the expected diamagnetism; diamagnetic currents are induced in the injected plasma in a sense so as to exclude the static flux (in accordance with Lenz's law). Note also that there is very little magnetic field in the poloidal cross section. This is to be expected since there are no spheromak currents in the injected plasma.

These data provide the basis for an explanation of the antialignment of the spheromak magnetic moment in the previous data set. Naively, one might expect that the currents and magnetic fields of a spheromak should behave like a rigid loop of current-carrying wire when injected into a magnetic field, viz., the magnetic moment of the loop should align with the magnetic field. However, in the case of established currents flowing in a plasma there are two effects upon injection into a static field. First, diamagnetic currents flow in a direction so as to reduce the static field on axis. Next, the preexisting current structure of the spheromak must orient itself in the magnetic system consisting of the static magnetic field and the opposing magnetic field due to the diamagnetic current. In other words, the spheromak, because it is a ball of dense plasma, digs a hole in the static magnetic field by excluding flux via diamagnetic currents. The spheromak currents can flow either with or against the diamagnetic current; it appears experimentally that the spheromak currents flow with the diamagnetic currents. The effect of this is that the diamagnetic field (which is opposed to the static field) and the spheromak field are aligned, which results in the observed field reversal on axis.

Another possible explanation of antialignment comes from considering the equation governing a force-free state, $\nabla \times \mathbf{B}=\lambda \mathbf{B}$ [see Eq. (1)]. As we noted in Sec. II A, the parameter $\lambda$ can be viewed as a geometrical quantity governed by the size of the system. The dimension $\lambda^{-1}$ can also be viewed as the scale in which the magnetic field must reverse direction in order to satisfy the force-free condition ${ }^{22}$ (this interpretation is particularly fruitful in the context of the reversed field pinch $^{13}$ ).

Note that before tilting to its final orientation, the spheromak in our experiment is in an axisymmetric, $m=0$ configuration (see the first frames in Fig. 6). We assert that when the spheromak merges with the dc toroidal field the total magnetic field that results must satisfy Eq. (1) locally. In other words, Eq. (1) must be satisfied when the fields have $m=0$ symmetry as it is later (after expanding and tilting) when the fields have $m=1$ symmetry.

The solution to Eq. (1) with $m=0$ symmetry is well known. ${ }^{6-8.13}$ Specifically,

$$
B_{z}=B_{0} J_{0}(y) \cos \left(k_{z} z\right)
$$

where $y=r\left(\lambda^{2}-k_{z}^{2}\right)^{1 / 2}, \lambda=\left(k_{r}^{2}+k_{z}^{2}\right)^{1 / 2}, k_{r}=3.83 / r$, and $k_{z}=\pi / L_{\mathrm{sph}}$. We see that $B_{z}$ (which is $B_{\mathrm{tor}}$ in toroidal geometry) reverses direction near the wall. This is the standard reversed field pinch solution but with finite $k_{z}$ (i.e., localized in toroidal extent). We have performed fits of the $m=0$ data using values of $\lambda$ and $k_{z}$ calculated with our 
geometry (estimating $L_{\mathrm{sph}}=4 r$ ). With no adjustable parameters (except the magnitude of $B_{0}$ ), we find good agreement with the finite $k_{z}, m=0$ model (better than $20 \%$ ). Note that if the spheromak had aligned with the dc toroidal field, then there would be no field reversal and the data would not fit the model at all. Note further that this explanation does not address the detailed dynamics of antialignment.

We conclude that in the case of $B_{\text {sph }}>B_{\text {tok }}$, the initial configuration of the spheromak is with its magnetic moment antialigned to the static toroidal ficld (often referred to as "bias field" in other contexts). This configuration is shortlived in our experiment as the spheromak rapidly tilts to the $m=1$ configuration. This result is not inconsistent with earlier observations of Turner $\mathrm{et} \mathrm{al}_{.}{ }^{23}$ They concluded "that in (their) geometry, axisymmetric CT's with bias flux opposite the poloidal flux on axis (i.e., antialigned) are stable only when the ratio $\psi_{b} / \psi_{\text {pol }}$ is less than $0.20-0.25$ " (where $\psi_{b}$ is the applied bias flux analogous to our applied toroidal field). If an acceleration stage is not employed, it is necessary that $B_{\mathrm{sph}}>B_{\mathrm{tok}}$ in order that the spheromak penetrate (transversely) across the applied field (see the discussion in Sec. $\mathrm{V})$. We therefore always operate in the regime $B_{\mathrm{sph}}>B_{\mathrm{tok}}$, where Turner et al. also observed antialignment of the spheromak.

On a few shots, we were able to initiate a tokamak discharge with the linear array inserted. The linear probe array was highly perturbative to tokamak operation. The maximum current we were able to attain with the probe inserted was about $1 \mathrm{kA}$ (without the probe the plasma current was 2-5 kA). In Fig. 9, we present data from such a shot. In Fig. 9(a), we subtract out the applied toroidal field (so $\widetilde{B}_{\text {tor }}=B_{\text {tor }}-690 \mathrm{G}$ ). It appears that the spheromak enters
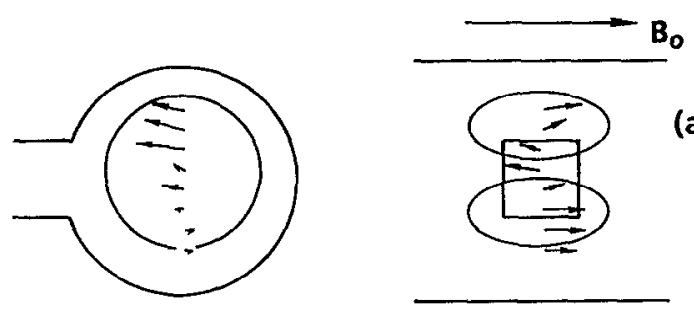

\section{$=690 \mathrm{G}$}

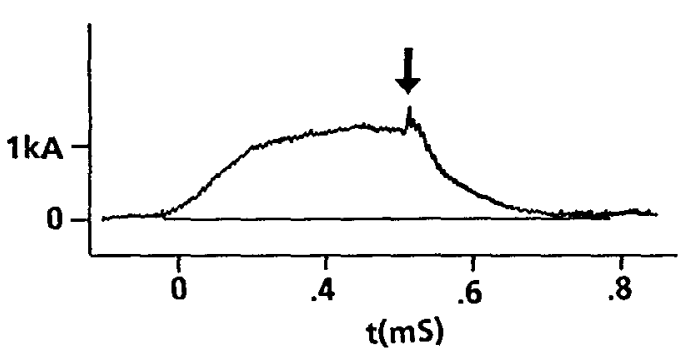

(b)

FIG. 9. Data from the linear array during spheromak injection into the tokamak plasma, left-handed spheromak injected into the left-handed tokamak. (a) Note that we observe spheromak antialignment with $B_{\text {tok }}$, we have subtracted the large dc component of the toroidal field for clarity; (b) a plot of tokamak plasma current. Spheromak fields depicted in (a) were obtained at the time indicated by the arrow. Note that we are only able to obtain a 1 $\mathrm{kA}$ discharge with the linear probe in place. the tokamak discharge with the previously observed antialignment (indicative of the first tilt). The spheromak remains in this configuration for the spheromak resistive lifetime; the second tilt appears to be suppressed. This shot produced the previously reported helicity injection effect ${ }^{1}$ [Fig. 9(b)], where the data in Fig. 9(a) were obtained during the tokamak shot at the time indicated by the arrow.

Note that the configuration in Fig. 9 is such that the spheromak toroidal field is opposed to the tokamak poloidal field (due to the tokamak plasma current). Nevertheless, we observe an enhancement of the tokamak plasma current because the spheromak and tokamak helicities add. This is troubling but is analogous to the observation of "flipped" spheromaks on the CTX experiment discussed in Barnes et al. ${ }^{15} \mathrm{~A}$ "flipped" spheromak is one in which the spheromak poloidal field on axis is directed opposite that of the stuffing flux on axis (see Barnes et al., Fig. 9). When the CTX spheromak is being sustained, current flows from the gun into the spheromak along field lines. However, when the spheromak is "flipped" the interpretation of current flow along field lines is problematic. To quote Barnes et al., "it is impossible in this case to construct an axisymmetric picture of sustainment by just current drive from one coaxial source electrode to the other along the outer flux surfaces." In the same way, interpretation of current drive by addition of spheromak toroidal flux to tokamak poloidal flux breaks down in our case. The interpretation that is consistent in both the CTX experiment and the present experiment is that of helicity injection and conservation. Current is driven in the CTX spheromak via helicity balance between gun and spheromak. Current drive by helicity injection and relaxation in tokamaks has been noted in other contexts. ${ }^{24,25}$ In our case, ${ }^{1}$ tokamak current is driven by the helicity balance between the injected spheromak and the tokamak discharge.

\section{B. Poloidal array results \\ 1. Quartz cross}

In order to ascertain more about the toroidal extent of the spheromak in the tokamak vessel a pair of quartz-jacketed, cross-shaped magnetic probe arrays were installed at locations immediately to the left and right of the spheromak entrance port (Fig. 2). We have measured the toroidal component of the spheromak magnetic field (in the tokamak frame) at four poloidal angles and three radii at both toroidal locations. We have also measured both the toroidal and poloidal components on one array (left or right). Each leg of the probe consists of three pairs of loops separated by $4 \mathrm{~cm}$ wound on Delrin forms. The forms are covered with a 0.001 in. slit brass electrostatic shield, precisely located in the 12 $\mathrm{mm}$ cross-shaped quartz jacket, and glued in place with high vacuum epoxy.

In Figs. 10-12 we show typical results of these measurements. The left (right) figure is data from the left (right) cross. The left side of each figure is the outboard side of the tokamak vessel, the right is the inboard. The diameter of each small circle represents the magnitude of the measured magnetic field while a dot (cross) in the center represents right-going (left-going) magnetic field. The scale size for the 
circles is the same for Figs. 10-12. The data presented are each from a single, unaveraged shot and time is measured from the arrival of spheromak magnetic fields at the probe array.

In Fig. 10, we present data from the quartz cross arrays with an applied, de toroidal field ( $170 \mathrm{G}$ on axis). Note that early after arrival, the spheromak fields are apparent on one array only. In the case of a right-going static field [applied field directed out of the page; Fig. 10(a) ], the spheromak appears initially at the left cross while the magnetic fields on the right cross depict primarily the vacuum toroidal field of the tokamak (note the largely undisturbed $1 / R$ variation from inboard to outboard). The largest circles represent about $400 \mathrm{G}$. At these early times, the spheromak has $m=0$ symmetry, where, again, the spheromak magnetic moment is antialigned with the static toroidal field (i.e., the spheromak fields reverse the direction of the applied field on the tokamak axis). For the case of left-going static field [Fig. $10(\mathrm{~b})]$, the spheromak fields are first observed on the right array. We conclude that there is a tendency for the spheromak (once it is embedded in the tokamak toroidal fields) to shift toroidally in the direction opposite that of the toroidal field. At present, we have no explanation for this phenomenon.

It appears that these large quartz arrays are a significant perturbation to the spheromak. The signals are smaller and spheromak lifetimes are shorter with the quartz crosses than

(a)

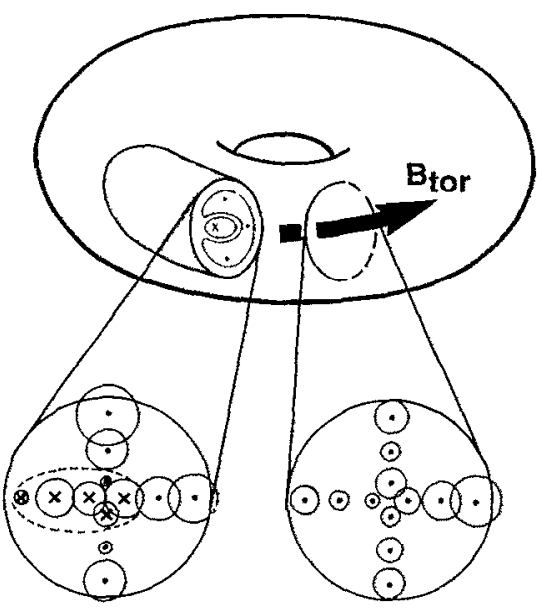

Btor Right, Shift Left

(b)
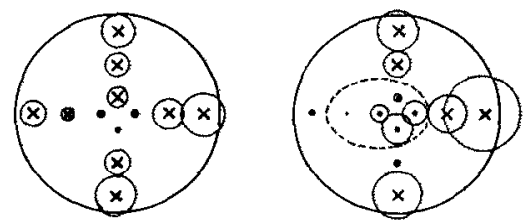

Btor Left, Shift Right

FIG. 10. Quartz cross data in the B field showing horizontal shift. (a) Right-going $170 \mathrm{G}$ applied field, spheromak fields shifted left, largest circle represents $375 \mathrm{G}, I_{\mathrm{gun}}=100 \mathrm{kA}, \Phi_{\mathrm{gan}}=0.4 \mathrm{mWb}, t=2 \mu \mathrm{sec}$ after arrival. (b) Left-going $170 \mathrm{G}$ applied field, spheromak fields shifted right, largest circle represents $440 \mathrm{G}, I_{\text {gun }}=75 \mathrm{kA}, \Phi_{\text {gur }}=0.3 \mathrm{mWb}, t=6 \mu \mathrm{sec}$ after arrival. with the smaller linear array. Particularly in the case of nonzero applied $B_{\text {tor }}$, there is evidence that the large array prematurely terminates the discharge and that the spheromak toroidal extent is localized or limited to the region immediately in front of the gun (between the quartz crosses). We seldom observe the transition from $m=0$ to $m=1$ symmetry with the quartz arrays (in only about $10 \%$ of the shots is there clear evidence of the transition). Evidently, the linear array was an acceptable perturbation to the spheromak discharge but the quartz array was marginal (with the most pronounced effect present when the static $B_{\text {tor }}$ is applied). In this regard, we may interpret the quartz crosses as end cap or surface probes, insofar as results appear to be from the extreme ends of the spheromak discharge.

We have found that spheromaks injected into the empty tokamak vessel without applied dc toroidal field provide more easily interpreted results. We see more signal indicating that the spheromak has some toroidal extent beyond the location of the crosses. We also readily observe the transition from $m=0$ to $m=1$ symmetry. Figure 11(a) shows the formation of a left-handed, $m=1$ equilibrium with similar signatures on the left and right arrays. Again, the data presented are from a single, unaveraged spheromak discharge (the circles represent actual data and not a fit to a model). The dashed lines are poloidal flux surfaces computed from our model. In Fig. 11 (b), we see that $10 \mu \mathrm{sec}$ later, the $m=1$

(a)

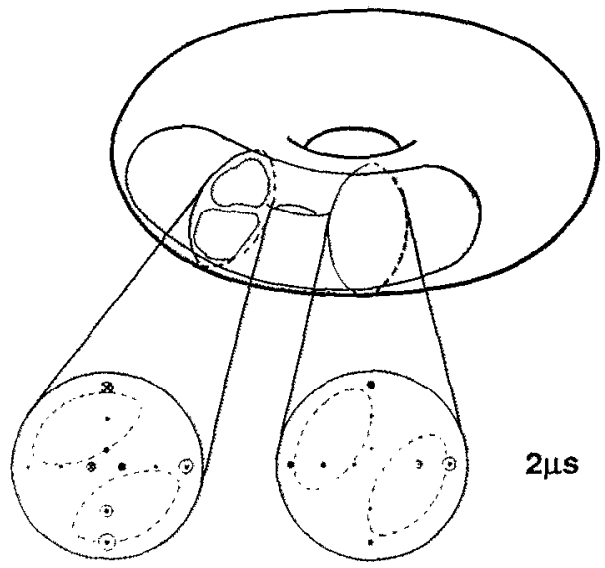

(b)
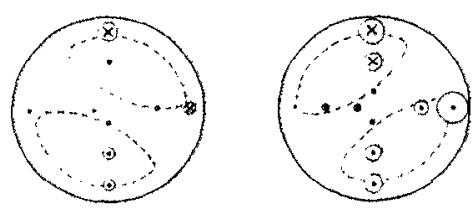

$11 \mu s$

(c)
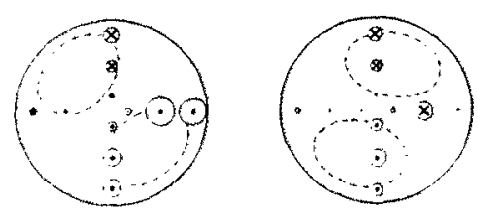

RIGHT HANDED

FIG. 11. Quartz cross data without net applied toroidal flux (and no tokamak plasma), outboard is to the left, inboard to the right. Largest circles represent $200 \mathrm{G}$. (a) Left-handed spheromak with $m=1$ symmetry in toroidal vessel at $2 \mu \mathrm{sec}$ develops. (b) A $60^{\circ}$ helical twist $(11 \mu \mathrm{sec})$; (c) A right-handed spheromak develops a twist with the opposite pitch. Dashed lines represent poloidal flux contours calculated from a fit of the data to a model. 
equilibrium has developed a pronounced $60^{\circ}$ helical twist. The data on the right array has the same form as on the left but rotated in the counterclockwise sense. The helical twist has been predicted by Schaffer. ${ }^{8}$

We have fit the data in Fig. 11 to our simple equilibrium model for an $m=1$, "double-helix," $\beta=0$, spheromak in an infinite cylindrical flux conserver (discussed in Sec. II B) and the data fits the model to within about $15 \%$. As an example, the relatively untwisted spheromak depicted in Fig. 11 (a) is fit to within $14.5 \%$ with $\lambda=24.4 \mathrm{~m}^{-1}$ and $k_{\mathrm{z}}=-2.3 \mathrm{~m}^{-1}$. The established, twisted spheromak of Fig. $11(b)$ is fit to within $11.5 \%$ with $\lambda=22.3 \mathrm{~m}^{-1}$. Note that the lower value of $\lambda$ at the later time is consistent with the expected relaxation process wherein magnetic energy is dissipated more rapidly than helicity. Recall that $\lambda_{\text {gun }}$, the value of this parameter at the spheromak's inception, was about $240 \mathrm{~m}^{-1}$. Based on the size of the cylindrical entrance region, we can estimate $\lambda_{\text {ent }} \cong 100 \mathrm{~m}^{-1}$. We can see that at each stage, the spheromak relaxes to an ever lower value of $\lambda$, consistent with lowering magnetic energy relative to helicity and increasing its physical size. The value of $k_{z}$ has increased to $-4.3 \mathrm{~m}^{-1}$ in Fig. 11 (b), indicative of an increased twist. We have calculated the magnetic energy of the spheromak, $W_{\text {mag }}=\left(1 / 2 \mu_{0}\right) \int B^{2} d^{3} x$ from the model, and we find for the equilibrium depicted in Fig. 11(b), $W_{\text {mag }}=0.675 \mathrm{~J}$. From the relationship $\lambda-2 \mu_{0} W_{\text {mag }} / K_{\mathrm{sph}}$, we can then calculate the spheromak helicity from the data fit and find $K_{\mathrm{sph}}=0.76 \times 10^{-7} \mathrm{~Wb}^{2}$

In Fig. 11(c), we show the formation of a right-handed, $m=1$ spheromak equilibrium [a different discharge from that depicted in Figs. 11 (a) and 11(b) formed by reversing the direction of the stuffing flux in the gun ]. Note that in this case, the twist is in the clockwise sense (about $60^{\circ}$ ) [opposite that of the left-handed spheromak of Figs. 11(a) and $11(b)]$. The fit of this discharge to out model has $k_{\mathrm{z}}=+4.6 \mathrm{~m}^{-1}$, about the same magnitude of that for the left-handed spheromak of Fig. 11(b), but of the opposite sign. The right-handed spheromak of Fig. 11(c) has the opposite sign of $\lambda$ of the left-handed spheromak of Figs. 11(a) and 11 (b) and, as predicted in Sec. II B, also has the opposite sign of $k_{z}$.

We are able to dedicate all our 24 channels of digitizers to a single cross (either left or right). In this experiment, we measure both the toroidal and poloidal components of the spheromak field (in the tokamak frame). In Fig. 12, we present data from two different shots (at roughly the same time during the discharge) with no net applied toroidal flux. The figure on the left represents data obtained from the left cross (both toroidal and poloidal field) while the figure on the right represents data from the right cross (from a different shot). Two interesting features are evident from these data. First, the spheromak's toroidal field (represented by arrows, the longest of which is $160 \mathrm{G}$ ) is clearly evident in both the left and right crosses. The spheromak toroidal field is directed downward on the left cross and upward on the right; this is evidently the spheromak toroidal field wrapping around its ends. Note that if this discharge were viewed from the spheromak gun, we would observe the same counterclockwise oriented spheromak toroidal field that was observed (a)

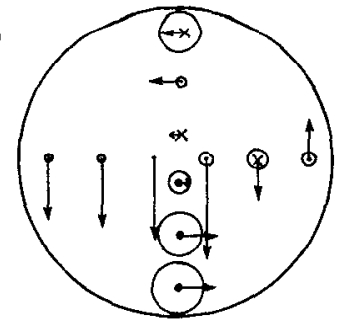

Left cross (b)

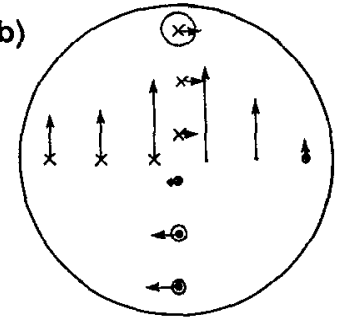

Right cross
FIG. 12. One side of a quartz cross array showing both toroidal and poloidal fields, no applied toroidal field. Here $I_{\text {gun }}=110 \mathrm{kA}, \Phi_{\mathrm{gun}}=0.4 \mathrm{mWb}$. The largest circles represent $200 \mathrm{G}$, the longest arrows represent $170 \mathrm{G}$. (a) A left cross depicting return toroidal flux (downward arrows), note close proximity of flux tubes on the inboard side of the tokamak, $t=8 \mu \mathrm{sec}$. (b) A right cross depicting return toroidal flux (upward arrows), $t=11 \mu \mathrm{sec}$.

with the linear array in Fig. 6. This vertical signature was not evident on the linear probe in the center of the discharge. This is strong evidence that the spheromak's extent toroidally around the tokamak is limited to the region roughly bounded by the crosses. It is from measurements of this sort that we have made the estimate that the length of the spheromak (its axial extent in the tokamak toroidal direction) $L_{\mathrm{sph}} \cong 4 r$ (where $r=12 \mathrm{~cm}$ is the tokamak minor radius).

Second, on the inboard side of the left cross there is evidence of two tubes of flux of opposite sign in close proximity. This small deviation from $m=1$ symmetry is always observed on the inboard side of the tokamak and is presumably a toroidal effect. The toroidal data (represented by the circles, the largest of which is about $240 \mathrm{G}$ ) shows the basic $m=1$ symmetry of the equilibrium.

\section{Eight-sided array}

With measurements at only four poloidal angles, we were unable to adequately resolve higher-order structure. An eight-sided probe array was used to obtain more information about the spheromak in a poloidal cross section (see Fig. 2). Loops were wound on $0.5 \mathrm{~cm}$ Delrin stock and coated with a thin $\left(0.005\right.$ in.) layer of ceramic paint ${ }^{26}$ (no quartz or electrostatic shield was used in order to minimize the size of the array). Active integrators with high common mode rejection were used in lieu of electrostatic shielding.

Figure 13 shows a typical data set from the eight-legged array with no net applied toroidal bias flux. In Fig. 13(a), we present raw data from the 16 outermost loops (the innermost eight were disabled for this shot) at about $10 \mu \mathrm{sec}$ after spheromak arrival ( $24 \mu \mathrm{sec}$ after the gun has been fired). In Fig. 13(b) we present the fit to this data and in Fig. 13(c) the difference between the data and the fit (actually $\mid$ data $\mid-$ |fit $\mid$ ) with the scale enlarged by a factor of 3 . A number of interesting features are brought out by this analysis. Note first that the $m=1$ shape crudely determined with the foursided quartz probe is verified. The least squares fit of the data to the simple infinite cylinder model is $11.4 \%$ with $\lambda=22.4 \mathrm{~m}^{-1}$. Second, it is clear from the data (as well as the plot of difference between data and cylindrical fit) that there is higher toroidal flux density on the inboard side of the 
(a)
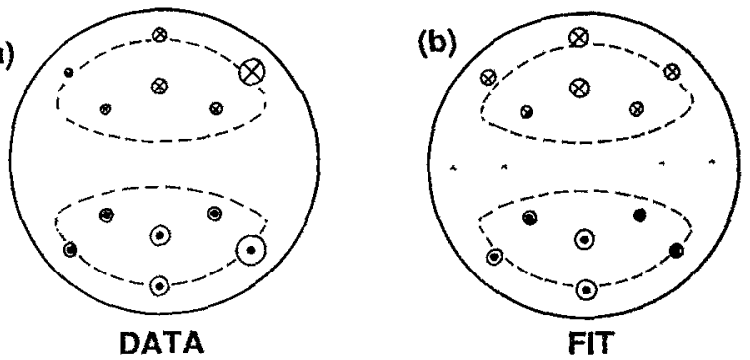

(c)

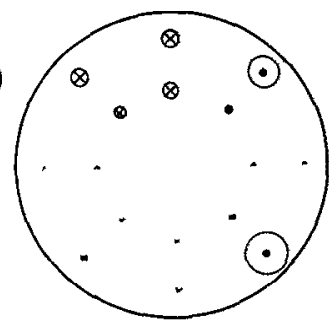

$3 \times(|D A T A|-|F| T \mid)$

FIG. 13. Eight-sided array. (a) Raw data from the eight-sided array; note the deviation from $m=1$ symmetry on the inboard side of the tokamak; the largest circles represent $200 \mathrm{G}, t=14 \mu \mathrm{sec}$. (b) The best fit of data to simple cylindrical model. (c) The difference between data and fit (scale increased by factor of 3 ); note the change of sign of error from inboard to outboard of tokamak and note that the fit is worse at larger radii.

tokamak than on the outboard side. Evidently, this small deviation from a pure $m=1$ symmetry is a toroidal effect not included in the cylindrical theory. Third, it appears that the simple model breaks down near the wall of the spheromak vessel. These deviations from the model (at larger radii ) may be due to nonuniform $J / B$ profile ${ }^{14}$ and finite $\beta$ effects. ${ }^{27}$ Work is proceeding on a more sophisticated model to address these deviations from the simple model.

\section{SPHEROMAK DYNAMICS IN THE TOKAMAK FIELD}

We have observed a number of phenomena that have a straightforward interpretation based on existing theories. The fact that axisymmetric spheromaks are unstable to a tilt if they become longer than they are wide is well established. In addition, the fact that spheromaks of opposite helicity sign should have opposite sense of twist and the appearance of toroidal effects in a tokamak vessel have direct interpretations. Observations of these phenomena have been presented in preceding sections. However, in this section, we present observations of spheromak dynamics in the tokamak geometry wherein the interpretation is not as direct (in fact, some of the observations are in conflict with present theories). First, we have observed and will discuss spheromak antialignment of its magnetic moment with the applied external field. Second, we present evidence that the spheromak does not penetrate into the tokamak fields unless $B_{\mathrm{sph}}>B_{\mathrm{tok}}$. Third, we discuss whether internal MHD or external forces dominate the dynamics of the spheromak. Finally, we address the relevance of the predicted Alfvén wake drag mechanism $^{3}$ in our experiment.

As was discussed earlier (Sec. IV A), we observe the $m=0$ spheromak configuration antialigned with the tokamak toroidal field (before ultimately tilting to the $m=1$

configuration ). Perkins et $a l^{4}$ have predicted that the spheromak magnetic moment becomes aligned with the tokamak field as a result of external torques (see Perkins et al., Fig. 1). The model used is that of a rigid conductor (analogous to a galvanometer coil) under the influence of torques arising from $J_{\text {sph }} \times B_{\text {tok }}$ forces. In this model, magnetic moment alignment is the energetically favored state.

It is evident from our experiments that spheromaks do not behave as one would expect a rigid, current-carrying conductor to behave. We have never observed alignment of our spheromak magnetic moments with the applied toroidal field. As was presented in Fig. 6, we observed the spheromak to be transiently antialigned with the tokamak toroidal field (before ultimately tilting to the lowest energy $m=1$ configuration). As we noted in Sec. IV A, this transient antialignment could be a diamagnetic effect or related to the spheromak behaving like a reversed field pinch with finite $k_{z}$.

Each of the theories relevant to spheromak injection into tokamaks ${ }^{3,4}$ has predicted that the kinetic energy density of the spheromak, $\rho v_{\mathrm{sph}}^{2} / 2$, must exceed the magnetic energy density of the tokamak $B_{\text {tok }}^{2} / 2 \mu_{0}$ in order that the spheromak penetrate the tokamak fields. This prediction is based on the simple physics of an object (the spheromak) in a conservative potential (the $1 / R$ variation of the tokamak toroidal field with major radius). In our case, $v_{\mathrm{sph}}$ is just the spheromak Alfvén speed (since we do not employ a separate acceleration stage) so the above condition reduces to $B_{\mathrm{sph}}>B_{\mathrm{tak}}$. In Fig. 14, we plot the peak fluctuating magnetic field of the spheromak equilibrium measured by the linear probe array as a function of the applied dc tokamak toroidal field. Note that the peak spheromak field at first increases (from $\sim 200 G$ when $B_{\text {tor }}=0$ ) as $B_{\text {tor }}$ is increased. This is because as the spheromak moves in the tokamak fields, magnetic flux is compressed against the flux conserving wall of the tokamak. At larger tokamak fields, the measured spheromak field decreases with increasing $B_{\text {tok }}$, suggesting that

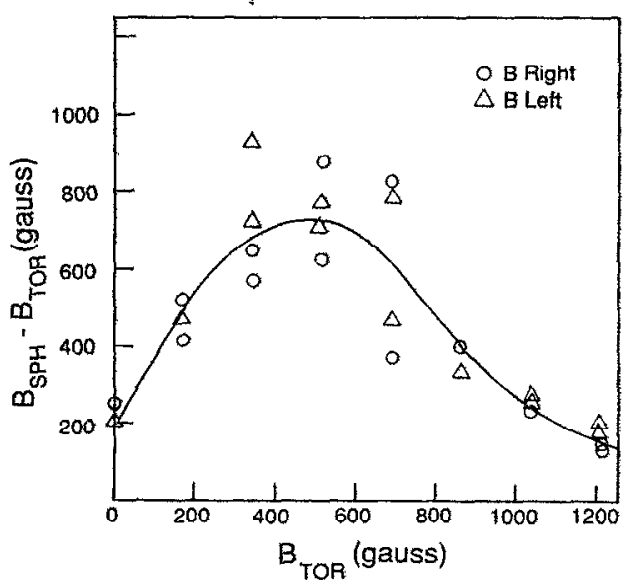

FIG. 14. Plot of peak measured $\widetilde{B}$ vs $B_{1, r}$. Note that above $B_{\mathrm{rtur}}=1000 \mathrm{G}$ we no longer observe spheromak fields in the tokamak vessel, indicating that the spheromak was stopped in the entrance region. The increase in signal at low $B_{1, t r}$ is due to compression of the applied toroidal flux by motion of the spheromak. Note that there is no dependence of this effect on direction of the applied field. 
the spheromak does not fully penetrate to the tokamak magnetic axis. The transition between the two regimes occurs when $B_{\text {tok }}$ is about equal to $B_{\mathrm{sph}}$ just as the spheromak enters the tokamak chamber.

From the standpoint of spheromak merging with the tokamak, it is important to ascertain whether the dynamics is governed by an interaction between the spheromak and tokamak fields or by MHD within the spheromak. One could imagine that the external field might prevent the spheromak from tilting to its lowest energy configuration. However, it appears that in our experiment, the external torque resulting from the interaction of spheromak magnetic moment with tokamak fields ${ }^{4}$ is dominated by internal MHD tilting. This is expected if $B_{\mathrm{sph}}>B_{\mathrm{tok}}$, since the time scale for an internal MHD tilt is an internal Alfvén time $\left.\left(\sim B_{, \mathrm{ph}}\right)^{1}\right)$, whereas the time scale for an external torque varies according to $\sim\left(B_{\mathrm{sph}} B_{\mathrm{tok}}\right)^{-1 / 2}$. The above inequality is certainly valid while the spheromak is in the cylindrical entrance region (outside the tokamak vessel where $B_{\text {tok }} \cong 0$ ). If the spheromak is to fully penetrate the tokamak fields, the condition must also be valid while the spheromak is in the tokamak vessel. If the spheromak is accelerated externally before injection into the tokamak ${ }^{28}$ then $B_{\mathrm{sph}}$ need not be greater than $B_{t o k}$ (since the spheromak kinetic energy is provided by the acceleration circuit and not the spheromak internal fields). We also observe spheromak expansion along tokamak field lines, but it appears that pressure balance in our case is maintained by the walls and not the tokamak plasma. ${ }^{3.4}$

Finally, a mechanism for slowing spheromaks as they move into the tokamak discharge has been proposed by Parks. ${ }^{3}$ In Park's model, the directed kinetic energy of the spheromak is coupled into Alfvén waves that propagate away from the spheromak as a wake. The energy removed by the Alfvén wake serves to slow the spheromak's motion, ultimately stopping it in the tokamak plasma. It appears that the Alfvén wake drag mechanism is weak in our experiment [ the stopping time resulting from this effect is roughly $t_{\text {stop }} \cong t_{\text {AIf }}$ $\left(n_{\mathrm{sph}} / n_{\mathrm{tok}}\right)^{1 / 2}$, which is longer than a spheromak lifetime in our case]. Resistive damping effects may play a dominant role in our experiments.

\section{CONCLUSION}

In summary, spheromaks have been injected into a vacuum vessel of the Caltech ENCORE tokamak (without tokamak plasma). The spheromak is observed to tilt first while in a cylindrical entrance region between coaxial plasma gun and tokamak (emerging into the tokamak with its magnetic moment antialigned with the applied toroidal field) and then observed to tilt again while immersed in the tokamak toroidal field. Magnetic lifetimes of the spheromak (with $m=1$ symmetry with respect to the tokamak vessel) are about $30 \mu \mathrm{sec}$. Probe measurements indicate that the spheromak temperature is $7 \mathrm{eV}$ and density is about $10^{15}$ $\mathrm{cm}^{-3}$. The spheromak is also observed to drift vertically in the $\mathbf{J}_{\text {center }} \times \mathbf{B}_{\mathrm{rok}}$ direction. Measurements with probe arrays on either side of the spheromak entrance port indicate that the spheromak shifts horizontally in the direction opposite that of the dc toroidal field. There is evidence that the spheromak remains toroidally localized in the region dircetly in front of the gun (so that $L \cong 4 r$ ). The spheromak is also seen to develop a helical twist and exhibits small deviations from $m=1$ symmetry on the inboard side of the tokamak. The spheromak equilibrium is well fit by a simple cylindrical model.

\section{ACKNOWLEDGMENTS}

It is a pleasure to acknowledge the technical assistance of Frank Cosso and Larry Begay, as well as numerous enlightening discussions with Dr. Cris Barnes, Dr. Tom Jarboe, Dr. Juan Fernández, Dr. Fred Wysocki, and Dr. George Marklin at Los Alamos National Laboratory and Dr. Charles Hartman and Dr. Jim Hammer at Lawrence Livermore National Laboratory.

This work was performed under U.S. Department of Energy Grant No. DE-FG03-86ER53232.

\section{APPENDIX: ENTRANCE REGION MEASUREMENTS}

In order to learn more about the formation of our spheromak for injection experiments we have measured the magnetic fields directly in front of the magnetized plasma gun. A test facility was constructed so that spheromaks could be formed in a cylindrical copper flux conserver with probe access at the end opposite the gun (Fig. 1). The facility is identical to the injection experiment up to the flange that mates with the tokamak port. The copper flux conserver (with same dimensions as the entrance region in the injection experiment) is $7.6 \mathrm{~cm}$ in diameter, $30.8 \mathrm{~cm}$ long, and has a $2 \mathrm{msec} L / R$ time (long compared to the spheromak lifetime).

In Fig. 15, we present data obtained in a cross section 20 $\mathrm{cm}$ from the gun muzzle. In this figure, we adopt the conventional cylindrical coordinates, where $z$ is directed away from the gun and $\phi$ is the azimuthal coordinate. Both the axial $\left(B_{z}\right)$ and azimuthal $\left(B_{\phi}\right)$ magnetic fields are measured at four radial positions, separated by $1 \mathrm{~cm}$. The magnetic probe array consisted of four pairs of loops on an L-shaped form [see Fig. 1(b)]. A slit copper foil served as an electrostatic shield and the array was covered with a quartz jacket. The data were obtained in eight shots at azimuthal positions separated by $\pi / 4$ (shots were highly reproducible near the gun). In this way we were able to fully map the magnetic structure of the spheromak in the region near the gun muzzle in situ. The $B_{z}$ data were fit with a low-order polynomial in $r$ and Fourier analyzed in $\phi$ (up to $m=4$ included) to generate a surface $B_{z}(r, \phi)$. Figure 15 (a) is a contour plot of the fit to $B_{z}$ as well as $B_{\phi}$, represented by arrows at a time $5 \mu \mathrm{sec}$ after spheromak formation ( $22 \mu \mathrm{sec}$ after the initiation of the discharge). Note that the basic magnetic shape has an $m=0$ symmetry (axisymmetric with the flux conserver) with an $m=2$ distortion. Near the axis of the flux conserver $B_{x}$ is directed away from the gun (out of the page), the magnetic axis is depicted by the heavier solid line and near the wall of the flux conserver the magnetic field is directed back toward the gun (dashed lines). The peak magnetic field is about $2100 \mathrm{G}$ on axis, the gun current was $90 \mathrm{kA}$ for this shot with $\Phi_{\text {gun }}=0.4 \mathrm{mWb}$. The plasma density is about $3 \times 10^{15}$ 

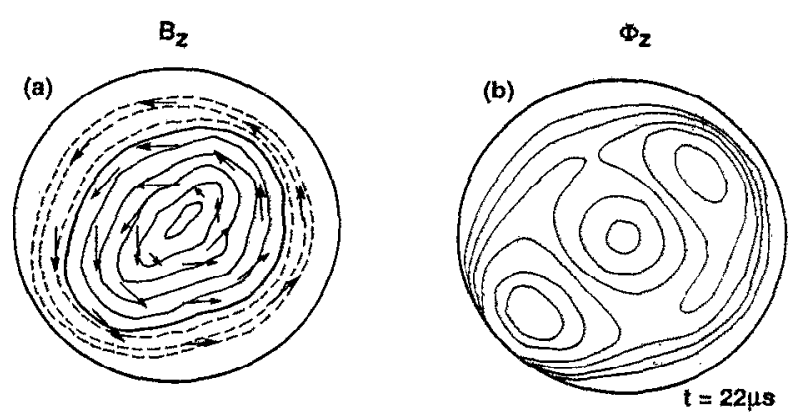

$$
\begin{aligned}
\operatorname{MAX} B_{z} & =2100 \mathrm{G} \\
\operatorname{MAX} \Phi_{z} & =.4 \mathrm{mWb} \\
f & =125 \mathrm{kHz}
\end{aligned}
$$
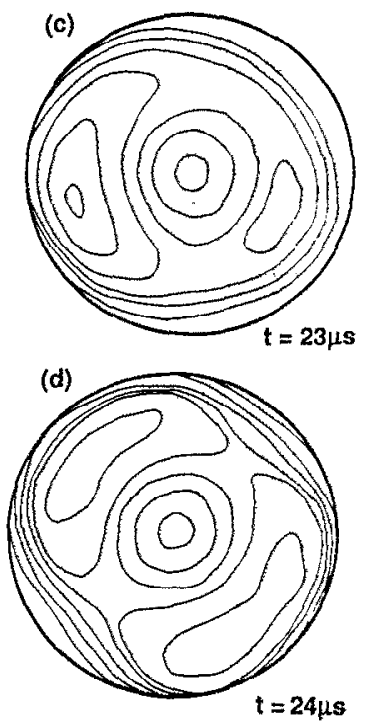

FIG. 15. Magnetic data from a cross section $20 \mathrm{~cm}$ in front of the gun. (a) Contours of axial magnetic field at $22 \mu \mathrm{sec}$ [solid (dashed) contours represent field directed away from (toward) the gun, peak field is $2100 \mathrm{G}$ (on axis), $400 \mathrm{G}$ per contour ], arrows show toroidal field. (b)-(d) The contours of axial flux at 22,23 , and $24 \mu \mathrm{sec}$ (the peak fiux is $0.4 \mathrm{mWb}$ and 0.08 mWb per contour).

$\mathrm{cm}^{-3}$ and $T_{e} \cong 10 \mathrm{eV}$. The Alfvén speed for these parameters is about $8 \times 10^{6} \mathrm{~cm} / \mathrm{sec}$. Figs. 15 (b) -15 (d) depict the axial flux at 22,23 , and $24 \mu \mathrm{sec}$. In this case, the axial magnetic field is integrated over the cross section, $\Phi_{z}(r)=2 \pi \int_{0}^{r} B_{z} r^{\prime} d r^{\prime}$. The $m=2$ distortion is more pronounced in this representation. The peak magnetic flux in the rotating lobes is about $0.4 \mathrm{mWb}$ (with each contour representing $0.08 \mathrm{mWb}$ ). Note that the analysis shows that magnetic flux is approximately conserved (the outcrmost contour being $\Phi_{z}=0$ ), indicating the reliability of the measurement and analysis. The rotation frequency is about 125 $\mathrm{kHz}$, corresponding to a rotational velocity of $3 \times 10^{6}$ $\mathrm{cm} / \mathrm{sec}$ (about $40 \%$ of the calculated Alfvén velocity).

Note that the mode rotates coherently (about $\frac{1}{4}$ cycle in $2 \mu \mathrm{sec}$ ) and the sense of rotation is in the $\mathbf{E} \times \mathbf{B}$ direction. Here $\mathbf{E}$ refer to the electric field produced by the gun voltage (directed radially outward) and $\mathbf{B}$ is the magnetic field resulting from the gun flux (directly axially away from the gun). The sense of rotation of the mode is also in the electron diamagnetic direction. The axial field near the wall $B_{z}$ (i.e., the spheromak poloidal field) is directed back toward the gun, while the pressure gradient near the wall is directed inward. Therefore the electron diamagnetic drift,
$v_{D e}=-\nabla p \times B / q n B,{ }^{2}$ is in the direction of the observed rotating mode.

Low azimuthal mode number magnetic oscillations are commonly observed in spheromak experiments. In the Los Alamos CTX experiment, $m=2$ oscillations are measured during the decay phase of the discharge. ${ }^{14}$ The oscillations are interpreted in terms of spatial variations in the parameter $J / B$ (where $J$ is the current density and $B$ is the magnetic field). According to the Taylor relaxation principle ${ }^{13}$ the magnetic fields of a spheromak obey the relation $\nabla \times \mathbf{B}=\lambda \mathbf{B}$ everywhere $(\lambda=$ const $)$. Since $\nabla \times \mathbf{B}=\mu_{0} \mathbf{J}$, this implies that a relaxed spheromak should have $J / B=$ const everywhere. If $J / B$ is indeed uniform, then the spheromak is stable, but if $J / B$ becomes smaller on the outer flux surfaces (as it does during the decay phase because cooler plasma becomes resistive on the outer surfaces), then the $m=2$ mode becomes unstable. The peaked $J / B$ profile results in increased magnetic shear and lower $q$, which is thought to drive an internal kink mode. The mode is observed to rotate in the electron diamagnetic direction in the CTX experiment.

In Sec. IV A, we inferred that the spheromak tilts from an $m=0$ configuration in the cylindrical entrance region to an $m=1$ configuration (see Fig. 6). However, we did not observe the tilt in the test facility. Instead, we observed the pronounced rotating $m=2$ mode followed by termination of the discharge after about $10 \mu \mathrm{sec}$. It is likely that unionized gas (from a slower valve than that used in the previously described experiments) prematurely terminated the discharge. The presence of a shroud of cool gas at the plasma edge (near the fux conserver wall) can cause a peaked $J / B$ profile as well as anomalous dissipation of helicity. ${ }^{29}$

'M. R. Brown and P. M. Bellan, Phys. Rev. Letl. 64, 2144 (1990).

${ }^{7}$ M. R. Brown and P. M. Bellan, Phys. Fluids B 2, 1306 (1990).

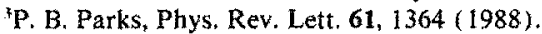

${ }^{+}$L. J. Perkins, S. K. Ho, and J. H. Hammer, Nucl. Fusion 28, 1365 (1988).

${ }^{5}$ T. R. Jarboe, I. Henins, H, W. Hoida, R. K. Linford, J. Marshall, D. A. Platts, and A. R. Sherwood, Phys. Rev. Lett. 45, 1264 (1980).

J. M. Finn, W. M. Manheimer, and E. Ott, Phys. Fluids 24, 1336 (1981).

${ }^{7}$ A. Bondeson, G. Marklin, Z. G. An, H. H. Chen, Y. C. Lee, and C. S. Liu, Phys. Fluids 24, 1682 (1981).

*M. J. Schaffer, Phys. Fluids 30, 160 (1987).

'J. C. Fernández, B. L. Wright, G. J. Marklin, D. A. Platts, and T. R. Jarboe, Phys. Fluids B 1, 1254 (1989).

${ }^{1}$ A. Janos, G. W. Hart, and M. Yamada, Phys. Rev. Lett. 55, 2868 ( 1985 ).

"M. N. Rosenbluth and M. N. Bussac, Nucl. Fusion 19, 489 (1979).

IJ. B. Taylor, Phys. Rev. Iett. 33, 1139 (1974).

${ }^{11}$ J. B. Taylor, Rev. Mod. Phys. 58, 741 (1986).

${ }^{14}$ S. O. Knox, C. W. Barnes, G. J. Marklin, T. R. Jarboe, I. Henins, H. W. Hoida, and B. L. Wright, Phys. Rev. Lett. 56, 842 (1986).

${ }^{15}$ C. W. Barnes, J. C. Fernández, I. Henins, H. W. Hoida. T. R. Jarboe, S. O. Knox, G. J, Markin, and K. F. McKenna, Phys. Fluids 29, 3415 (1986).

${ }^{1}$ H. Alfvén, L. Lindberg, and P. Mitlid, J. Nucl. Energy, Part C: Plasma Phys. 1, 116 (1960).

${ }^{17} \mathrm{C}$. W. Barnes, T. R. Jarboe, G. J. Marklin, S. O. Knox, and I. Henins, Phys. Fluids B 2, 1871 (1990).

${ }^{18}$ Kindly provided by I. Henins, Los Alamos National Laboratory.

${ }^{19}$ F. F. Chen, in Plasma Diagnostic Techniques, edited by R. H. Huddlestone and S. L. Leonard (Academic, New York, 1965), p. 113.

${ }^{20}$ F. F. Chen, Introduction to Plasma Physics (Plenum, New York, 1974), p. 184.

${ }^{21}$ A. W. Leonard, R. N. Dexter, and J. C. Sprott, Phys. Rev. Lett. 57, 333 (1986).

${ }^{22}$ T. R. Jarboe (private communication).

${ }^{23}$ W. C. Turner, G. C. Goldenbaum, E. H. A. Granneman, J. H. Hammer, 
C. W. Hartman, D. S. Prono, and J. Taska, Phys. Fluids 26, 1965 (1983). ${ }^{24}$ M. Ono, G. J. Green, D. Darrow, C. Forest, H. Park, and T. H. Stix, Phys. Rev. Lett. 59, 2165 (1987).

${ }^{25}$ T. R. Jarboe, Fusion Technol. 15, 7 (1989).

${ }^{26}$ A. Janos, G. W. Hart, C. H. Nam, and M. Yamada, Phys. Fluids 28, 3667 (1985).
${ }^{27}$ G. W. Hart, C. Chin-Fatt, A. W. DeSilva, G. C. Goldenbaum, R. Hess, and R. S. Shaw, Phys. Rev. Lett. 51, 1558 (1983).

${ }^{28}$ C. W. Hartman and J. H. Hammer, Phys. Rev. Lett. 48, 929 (1982).

${ }^{29}$ J. C. Fernández, C. W. Barnes, T. R. Jarboe, I. Henins, H. W. Hoida, P. L. Klinger, S. O. Knox, G. J. Marklin, and B. L. Wright, Nucl. Fusion 28, 1555 (1988). 